\title{
THE EFFECT OF ASYMMETRY ON MICROMIXING IN CURVILINEAR MICROCHANNELS
}

\author{
SARP AKGÖNÜL \\ Submitted to the Graduate School of Engineering and Natural Sciences \\ in partial fulfillment of \\ the requirements for the degree of \\ Master of Science
}

SABANCI UNIVERSITY

July 2018 


\section{THE EFFECT OF ASYMMETRY ON MICROMIXING IN CURVILINEAR MICROCHANNELS}

\section{APPROVED BY:}

Prof. Dr. Ali Koşar

(Thesis Supeivisor)

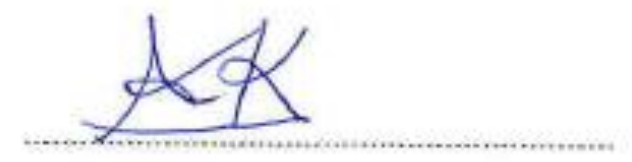

Asst. Prof. Dr. Yegân Erdem

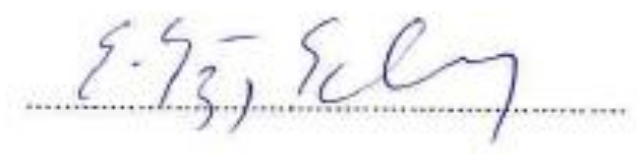

Assoc. Prof. Di, Özlem Kutlu

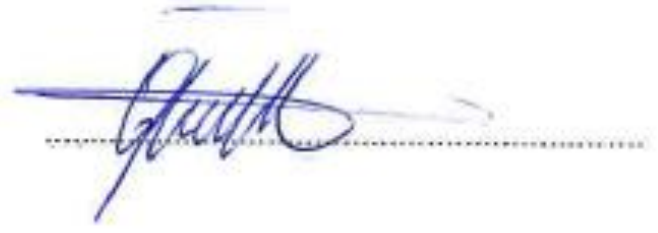

DATE OF APPROVAL: $\quad$ 20/07/2018 
(C) Sarp Akgönül 2018

All Rights Reserved 


\title{
THE EFFECT OF ASYMMETRY ON MICROMIXING IN CURVILINEAR MICROCHANNELS
}

\author{
Sarp Akgönül \\ Mechatronics Engineering, M.Sc. Thesis, 2018 \\ Thesis Supervisor: Prof. Dr. Ali KOŞAR
}

Keywords: Passive mixing, chaotic advection, dean vortices, curvilinear PDMS microchannels, asymmetrical microchannels

\begin{abstract}
The necessity of microscale mixing processes has been tremendously increasing in most of the microsize chemical and biochemical devices during recent years, particularly in the design of lab-on-a-chip and micro-total analysis systems. Different approaches were implemented in the available micromixers in the literature for improving the mixing performance.

Due to the absence of any external source, mixing by utilizing passive mixing techniques is more economical. In curvilinear microchannels, which offer effective passive mixing, chaotic advection results in continuous radial perforation of inter-diffusion layer between the fluid streams due to the transverse secondary flows. In this dissertation, the effects of Dean vortices and secondary flows were investigated in asymmetrical polydimethylsiloxane curvilinear rectangular microchannels, which were fabricated by onestep lithography process and had repeated S-shape patterns with a curvature of $280^{\circ}$ along the channel.
\end{abstract}


Moreover, the effect of asymmetry was assessed by comparing the mixing results with symmetrical microchannels. Mixing performance was analyzed by using $\mathrm{NaOH}$ and phenolphthalein solutions as mixing fluids, which entered from the channel inlets. According to the results, the significant effects of stretching and contracting motion of Dean vortices revealed themselves above a certain Dean number value, thereby making the asymmetrical microchannel outperform the symmetrical channel in the mixing performance. Below this threshold, the symmetrical microchannel was observed to be superior to the asymmetrical microchannel. 


\title{
DÖNEL MIKROKANALLARDA ASIMETRININ KARIŞMAYA ETKISII
}

\author{
Sarp Akgönül
}

Mekatronik Mühendisliği, Yüksek Lisans Tezi, 2018

Tez Danışmanı: Prof. Dr. Ali KOŞAR

\begin{abstract}
Anahtar kelimeler: Pasif karışma, kaotik adveksiyon, dean vortisleri, dönel PDMS mikrokanallar, asimetrik kanallar
\end{abstract}

\section{ÖZET}

Yakın dönem dikkate alındığında mikro boyutlu kimyasal ve biyokimyasal cihazların birçoğunda mikro-ölçek karıştırma işlemi ihtiyacında muazzam bir artış olmuştur. Bilhassa çip üstü laboratuvar ve mikro-total analiz sistemlerinin tasarımında bu ihtiyaç daha yoğundur.

Karışım performansını artırmak için literatürde mevcut mikromikserler içinde farklı yaklaşımlar uygulanmıştır. Herhangi bir harici kaynağın bulunmaması sebebiyle, pasif karıştırma teknikleri kullanımı karıştırma işlemini daha ekonomikleştirmektedir. Etkili pasif karıştırma sağlayan dönel mikrokanallarda ortaya çıkan kaotik adveksiyon, yatay düzlemde oluşan ikincil akışlara yol açarak akışkan akışları arasında inter-difüzyon tabakasının sürekli delinmesini sağlar. Bu tez çalışmasında, tek adımlı litografi işlemiyle imal edilen ve kanal boyunca $280^{\circ}$ 'lik bir eğrilik ile tekrarlanan S-şekil şablona sahip olan asimetrik polidimetilsiloksan (PDMS) dönel dikdörtgen mikrokanallarda, Dean girdaplarının ve ikincil akışların etkileri araştırılmıştır. 
Buna ek olarak, simetrik mikrokanallar ile karıştırma sonuçları karşılaştırılarak asimetrinin etkisi değerlendirilmiştir. Karıştırma performansı, karıştırma akışkanları olarak $\mathrm{NaOH}$ ve fenolftalein çözeltileri ile analiz edilmiştir. Elde edilen sonuçlara göre, Dean girdaplarının gerilme ve büzülme hareketinin efektif etkileri, belirli bir Dean sayı değerinin üzerin kendini ortaya koymuştur ve bu noktadan sonra asimetrik mikrokanalın karıştırma performansında simetrik kanaldan daha iyi bir performans gösterdiği gözlemlenmiştir. Bu değer eşiğinin altında olunduğunda ise simetrik mikrokanalın, asimetrik mikrokanala nazaran daha üstün bir karıştırma performansına sahip olduğu gözlemlenmiştir. 


\section{ACKNOWLEDGEMENT}

First and foremost, I wish to express my deepest gratitude to Prof. Dr. Ali Koşar for his support, guidance and encouragement. He saw beyond the false silhouette of incoherent grades and believed in me thus my graduate journey has begun. I sincerely believe that if I were to reach my ultimate goals, it will be thanks to the initial brick that Prof. Dr. Ali Koşar placed. I am and always will be honored to work with him.

I would like to express my appreciation towards my colleagues at the "Micro-Nano Scale Heat Transfer and Microfluidics Research group" where my colleagues Arzu Özbey and Mehrdad Karimzadehkhouei helped me to adapt into the graduate academic life and provided important contributions for the completion this project.

Abdolali Khalili Sadaghiani, has become my fellow traveler and my best friend which turned the fierce academic environment into a challenging but fun jungle to cross. I cannot imagine this period without his friendship and support. I will always treasure our time together. I am absolutely grateful to have known Mükerrem Ilker sevgen who had tremendous impacts on me related both academic life and social life. Considering all of my work throughout this journey, he had a part in every single one of them so I am thankful to have worked with such bright minds.

I would also want to emphasize the support of my family as they encouraged me and filled me with confidence I needed to overcome any difficulty that came across my way. My mother and my father believed in me so much that made me more and more driven. Special thanks to my sister, who is the secret hero that mold me into who I am today.

Finally, my beloved wife, Eda Şener Akgönül. The words cannot express what she means to me and how she effected my life. Every obstacle we faced we overcame together and I had the chance to always feel as a whole facing each challenge, thanks to her. I will cherish everything she did for me and hope to make her feel the same way. 


\section{TABLE OF CONTENTS}

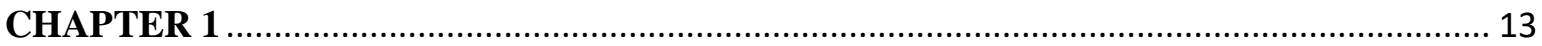

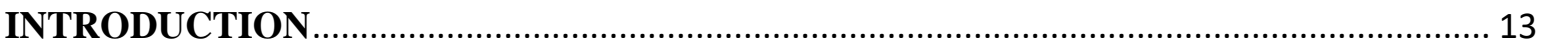

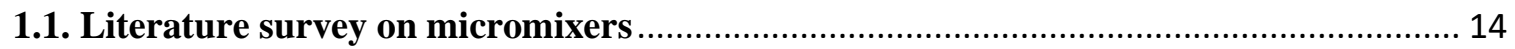

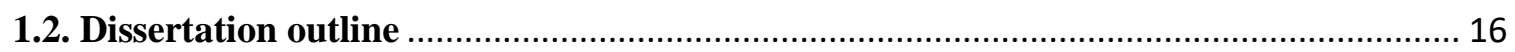

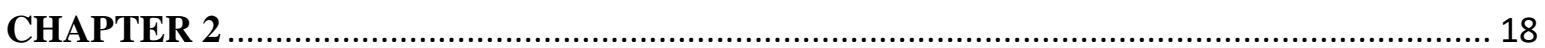

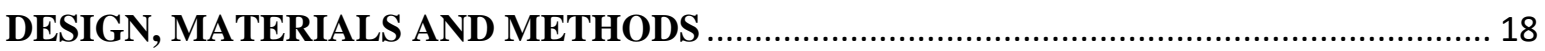

2.1 Micromixer Design and Working Principle

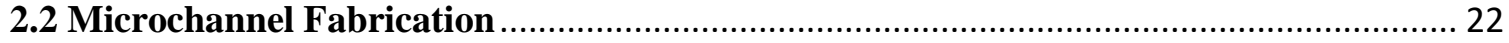

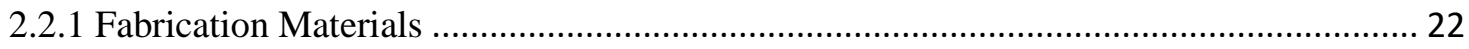

2.2.2 Fabrication Procedure ………………………………………………………... 24

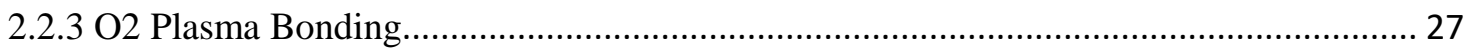

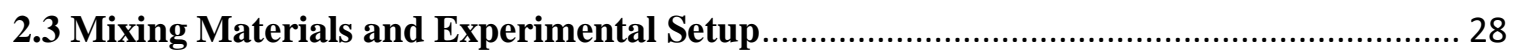

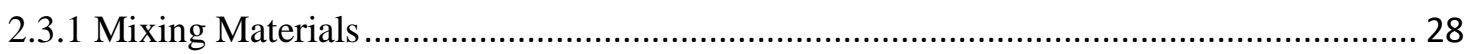

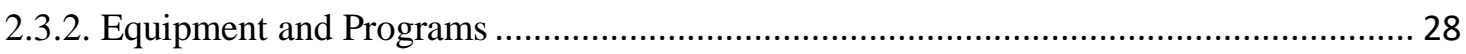

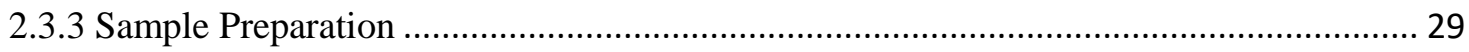

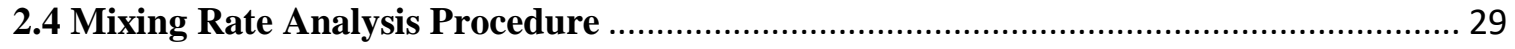

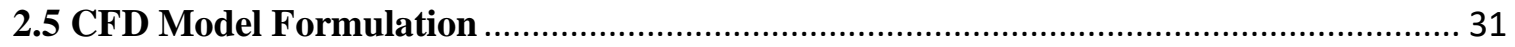

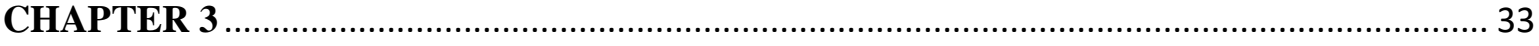

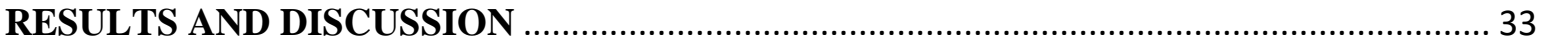

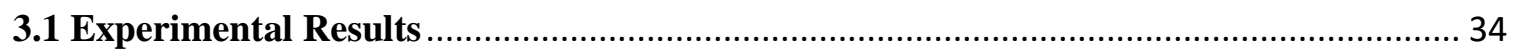

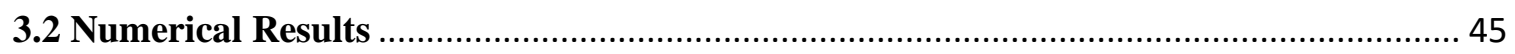

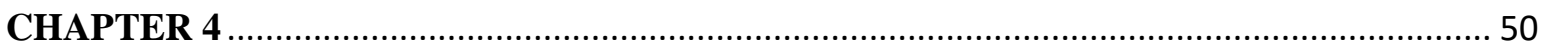

CONCLUSION AND FUTURE WORK ……………………………………………… 50

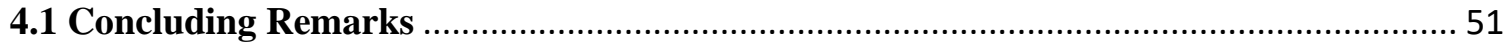

4.2 Future Work 


\section{LIST OF FIGURES}

Figure 1: Designs of a) asymmetrical micromixer having a varying width (450 $\mu \mathrm{m}$ to $600 \mu \mathrm{m})$ and b) symmetrical micromixer having a width of $450 \mu \mathrm{m}$, which demonstrate the geometry of the microchannels, the mixing index evaluation lines indicated as A-A, B-B, C-C, and the velocity profile lines indicated as S1, S2 and D-D. The blue dashed rectangles show the locations, where the images were taken. c) The image of the experimental device, which shows the aqueous solutions of sodium hydroxide $(\mathrm{NaOH})$ and Phenolphthalein (phph) introduced to the inlets (on the right) while depicting the resulting fuchsia color after the mixing process (on the left).

Figure 2: The 2D acetate mask designs of a) asymmetrical microchannel b) symmetrical microchannel

Figure 3: Film thickness vs spin speed chart of SU-8 3050 provided by MicroChem corporation 24

Figure 4: Illustration of the essential steps in PDMS microchannel fabrication process.....

Figure 5: The optimum mesh element structure generated for the computational study 31

Figure 6: Mixing images of Phenolphthalein and $\mathrm{NaOH}$ aqueous solutions in: i) asymmetrical and ii) symmetrical micromixers at the given Dean numbers. The visualization was performed at the entrance region, and the corresponding flow rates are a) $400 \mu \mathrm{l} / \mathrm{min}$, b) $1000 \mu \mathrm{l} / \mathrm{min}$, c) $2000 \mu \mathrm{l} / \mathrm{min}$, d) $2400 \mu \mathrm{l} / \mathrm{min}$, e) $3000 \mu \mathrm{l} / \mathrm{min}$. 35

Figure 7: Mixing images of Phenolphthalein and $\mathrm{NaOH}$ aqueous solutions in: i) asymmetrical and ii) symmetrical micromixers at the given Dean numbers. The visualization was performed at the sixth turn of the microchannel, and the corresponding flow rates are a) $400 \mu \mathrm{l} / \mathrm{min}$, b) $1000 \mu \mathrm{l} / \mathrm{min}$, c) 2000 $\mu 1 / \mathrm{min}$, d) $2400 \mu \mathrm{l} / \mathrm{min}$, e) $3000 \mu \mathrm{l} / \mathrm{min}$.

Figure 8: Mixing index as a function of Reynolds number (bottom x-axis) and Dean number (top xaxis) for symmetrical and asymmetrical micromixers at a) entrance b) sixth turn 39

Figure 9: Visual mixing data of Phenolphthalein and $\mathrm{NaOH}$ aqueous solutions in: i) asymmetrical and ii) symmetrical micromixers for the given Dean numbers below the specified critical value $(\mathrm{K}<16.8)$. The visualization was performed at the outlet region where corresponding flow rates are a) $100 \mu \mathrm{l} / \mathrm{min}$, b) $400 \mu \mathrm{l} / \mathrm{min}$, c) $700 \mu \mathrm{l} / \mathrm{min}$, d) $1000 \mu \mathrm{l} / \mathrm{min}$, e) $1300 \mu \mathrm{l} / \mathrm{min}$, f) $1600 \mu \mathrm{l} / \mathrm{min}$ and g) 1900 $\mu \mathrm{l} / \mathrm{min}$ 40

Figure 10: Mixing images of Phenolphthalein and $\mathrm{NaOH}$ aqueous solutions in: i) asymmetrical and ii) symmetrical micromixers for Dean numbers above the specified critical Dean number $(K>16.8)$. The visualization was performed at the outlet region and the corresponding flow rates are a) 2000 $\mu 1 / \mathrm{min}$, b) $2200 \mu \mathrm{l} / \mathrm{min}$, c) $2400 \mu \mathrm{l} / \mathrm{min}$, d) $2600 \mu \mathrm{l} / \mathrm{min}$, e) $2800 \mu 1 / \mathrm{min}$ and f) $3000 \mu 1 / \mathrm{min}$ 
Figure 11: Mixing index as a function of Reynolds number (bottom x-axis) and Dean number (top

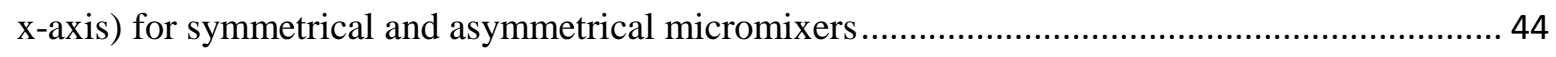

Figure 12: Velocity profiles of the asymmetrical microchannel near the outlet region showing the velocity profile shift after each inflection point at the flow rate of $2000 \mu \mathrm{l} / \mathrm{min}$, where the asymmetrical micromixer begins to outperform the symmetrical counterpart .45

Figure 13: Cross-sectional velocity profile of the stretching region with a width of $450 \mu \mathrm{m}$ (upper) and the contraction region with a width of $600 \mu \mathrm{m}$ (lower) and their corresponding Dean vortices at the flow rate of $2000 \mu \mathrm{l} / \mathrm{min}$ 46

Figure 14: Cross-sectional velocity profile at regions: a) C-C line (width of $450 \mu \mathrm{m}$ ) and b) D-D line (width of $600 \mu \mathrm{m}$ ), showing Dean vortices at the flow rate of $2000 \mu \mathrm{l} / \mathrm{min}$, which is the critical pointi beyond which the asymmetrical micromixer outperforms the symmetrical one ................... 48 


\section{LIST OF TABLES}

Table 1: The physical properties of SU-8 3050 provided by MicroChem corporation................... 22

Table 2: The material properties of PDMS provided by Dow corning corporation ......................... 23

Table 3: The process specifications of SU-8 3050 provided by MicroChem corporation ............... 25 
CHAPTER 1

INTRODUCTION 


\subsection{Literature survey on micromixers}

Since the emergence of miniaturized microfluidic devices, micromixing has become one of the major interdisciplinary subjects thanks to the integration of micro-total analysis systems ( $\mu \mathrm{TAS})$, which embody bio-analytical and chemical processes such as enzyme reaction, protein folding and drug delivery (Beebe, Mensing, and Walker 2002; Jeong et al. 2010). Besides the high and diverse potential of such microfluidic devices, the advantages of embedded micromixers come in sight with shorter mixing lengths and times combined with the use of small amounts of reagents. Nevertheless, to achieve such comprehensive devices, some limitations imposed by laminar flows in micro scale mixers must be overcome. Thus, the demand for optimization and amelioration of micromixer capabilities for efficient, high

throughput microfluidic devices with a comparably lower cost has been continuously growing (Kockmann et al. 2006).

Micromixers are divided into two main categories: passive and active (Lee et al. 2016) mixers. The absence or presence of an external disturbance or energy is the deciding factor. While active mixers operate via external power sources, passive mixers solely benefit from channel geometries (Nguyen and Wu 2005). Furthermore, active micromixers have a higher cost than passive micromixers due to energy consumption and relatively expensive fabrication methods (Ward and Fan 2015).

Both mixer types have their own set of sub-categories. Sub-categories of passive mixers relying mainly on diffusion or advection are reported as parallel lamination, serial lamination, droplet, injection and chaotic advection (DeMello 2006). In passive mixers of parallel lamination, the initial streams of fluids are divided into $n$ substreams and are then combined at the intersection point (Cha et al. 2006; Koch et al. 1999), while the streams are subjected to recurrent separation and recombination of pathways (Afzal and Kim 2012; Xia et al. 2006) along the microchannel for serial lamination. Considering droplet mixers, they mostly have discrete flow regimes since droplets punctuated by air are generated and driven towards each other to generate the mixture (Liu et al. 2006; Voldman, Gray, and Schmidt 2000). In injection mixers, the aim is to spray the desired reagents through singular or multiple capillary nozzles into the solvent flow (Hosokawa, Fujii, and Endo 1999). A 
numerical study on the capillary based mixing with twisted 3D capillary tubes can also be found in the literature (Zhang, $\mathrm{Hu}$, and $\mathrm{Wu} 2012$ ).

The chaotic advection mixers, which is also the type of the micromixer in this study, benefits from the effect of geometrical manipulations exerted on the flow either by obstacles and patterns or repetitive sudden distortions in stream pathways, which results in stretching, folding or breaking of the fluid flow (Sudarsan and Ugaz 2006; Cook 2013). The basic emerging geometries are T-type, Y-type (Gobby, Angeli, and Gavriilidis 2001; Yi and Bau 2003), split and recombine types (Park et al. 2008; Schwesinger, Frank, and Wurmus 1999) for lamination based micromixers, whereas patterned surfaces (Kim et al. 2004), Zig-zag shaped (Mengeaud, Josserand, and Girault 2002), Tesla structured (Hong, Choi, and Ahn 2004) and serpentine (Park et al. 2008) types were considered for chaotic advection based micromixers. Serpentine type micromixers benefitting from chaotic advection provides simple structures with curvilinearity thereby offering a minimized possibility of clogging and shear damage onto the bio-samples (Kang et al. 2009). 3D structured microchannel geometries can provide complex trajectories and induce the folding and stretching motion on the fluid, which results in effective mixing (Singh et al. 2008; Xia et al. 2005). The secondary flows in chaotic mixing generated by obstacles, grooves or microchannel curvilinearity improves rapid mixing ability while providing simplicity in design and fabrication (Fan et al. 2017; Kang et al. 2008).

Particle tracing based numerical studies provided an insight into flows inside these micromixers (Aubin, Fletcher, and Xuereb 2005; Stroock and McGraw 2004), and new studies present new analysis methods such as the improved mapping method (Singh et al. 2008). In the literature, another mixing investigation methodology, chaotic analysis and fluorescent resonant-energy transfer (FRET) was presented for chaotic advection micromixers with grooves and split and recombine type micromixers (Y. T. Chen et al. 2011; Tung and Yang 2008).

The necessity of various recirculation patterns for a chaotic advection based high micromixing performance and the insufficiency of sole helical flows to generate chaotic mixing is emphasized in the literature (Hardt et al. 2005). In another study, polymer 
membranes were encapsulated benefitting from chaotic mixing (Gallé and Steinberg (Gallé and Steinberg 2016).

The governing mixing mechanism is also the main deciding factor, which needs to be reckoned. While flows with low Reynolds numbers combined with microchannels of relatively small characteristic lengths rely on diffusion between the mixing inter-layers, flows inside relatively larger microchannels with characteristic lengths of (100-1000 $\mu \mathrm{m})$, where higher Reynolds numbers are obtained, promote advection mixing rather than diffusion (Lee et al. 2016; DeMello 2006; Hardt et al. 2005). In order to fully comprehend the dynamics of mixing process, another crucial dimensionless number, Peclet number, which deserves attention, is employed and represents the domination of advection over diffusion. In the cases of low Peclet numbers coupled with low Reynolds numbers, mixing is dominated by molecular diffusion (Livak-Dahl, Sinn, and Burns 2011), while advection is dominant in mixing in the cases of high Peclet numbers coupled with high Reynolds numbers (Kockmann et al. 2006). Mixing becomes challenging for a mixing process with a high Peclet number and a low Reynolds number (Tofteberg et al. 2010).

The focus of this study is on chaotic advection and its effectiveness, which is accomplished with secondary flows transverse to the original fluid flow path generated by two counter-rotating vortices. In the literature, such secondary flows and the effect of chaotic advection in mixing were well inspected in symmetrical microchannels (Jiang et al. 2004). Due to the nature of this type of micromixing, an increase in interfaces between fluids is observed, which implies an increase in diffusion flux across interfaces in an exponential manner, substantially enhance mixing (Marques and Fernandes 2011). The folding and stretching of the streamline due to the transverse flows inside chaotic mixers pierce the diffusion layer between the reagents and promote rapid mixing (Fan et al. 2017; Ward and Fan 2015).

\subsection{Dissertation outline}

This dissertation presents a parametric micromixing study on curvilinear microchannels with asymmetrical and symmetrical configurations based on the width. Both micromixers presented in this dissertation were conformably fabricated using PDMS 
(polydimethylsiloxane) with the use of conventional soft lithography microfabrication methods. Another parameter, which was kept the same for both of the micromixers, is the number of inflection points appearing at each turn, which is 11 . While these mixers have the same curvature degree of $280^{\circ}$, inner diameter of $800 \mu \mathrm{m}$ (distance from the center of circumference of each turn to the relevant inner wall) and height of $\sim 88 \mu \mathrm{m}$, the difference lies in the width since the symmetrical microchannel has a width of $450 \mu \mathrm{m}$, and the asymmetrical one has a width repetitively varying between 450 and $600 \mu \mathrm{m}$. The interchanging width of an asymmetrical microchannel introduces a stretching and contracting motion over the transverse flows along the microchannel. The effect of such a motion on the mixing performance was investigated with this work. The purpose of the widening of the asymmetrical microchannel is to generate the effect of stretching of the Dean vortices on mixing. The more the vortices stretch the weaker they become. A widening asymmetrical microchannel benefits from the stretching and contracting motion. The vortices get weaker at each wider turn. As a result, this cyclic weakening and re-stabilizing of the Dean vortices are hypothesized to be more effective in mixing than regular vortices generated in a symmetrical microchannel. The possibility of having geometrical asymmetry in microchannels presents a broad lane for optimizing microchannel configurations. Moreover, the physics of the secondary flows, which form in chaotic advection mixers, is yet to be fully understood. Such secondary flows can be exploited for mixing, particle enrichment and isolation or bio-particle sorting. They do not necessarily have to be confined within a symmetrical geometry. The advantage of such curvilinear microchannels with serpentine, $\mathrm{C}$ shape or helical configurations in the applications of mixing or isolation of particles is that they reduce the possibility of shear damage on bio-samples compared to those of with obstacles, grooves or sharp edges (Kang et al. 2009).

In order to fully benefit from the advantage of rapid mixing in chaotic advection mixers with high mixing performance, comprehending secondary flows inside both symmetrical and asymmetrical micromixers is of prime importance. 


\section{CHAPTER 2}

DESIGN, MATERIALS AND METHODS 


\subsection{Micromixer Design and Working Principle}

The methodology of this study includes the comparison of the mixing performances of two curvilinear micromixers, one having a constant width and the other having a varying (asymmetrical) width after a turn. These micromixers benefitting from chaotic advection due to the curvilinear geometry are also considered as Dean-vortices based mixers. The effect of the curvilinearity resulting from the recurrently changing circular pathways (turns) with a curvature degree of $280^{\circ}$ reveals itself with transverse secondary flows leading to two counter-rotating vortices called Dean vortices inside both upper and bottom halves of the microchannel (Sudarsan and Ugaz 2006). As the radial circulation of these Dean vortices is directed towards the outer wall around the midline and in reciprocal direction near bottom and top planes of the micromixer, the inter-diffusion layer between the two reagents is continuously pierced in a complementing way by these opposing transverse flows. In order to quantify the magnitude of these counter-rotating vortices, a dimensionless number called Dean number $(K)$ is defined as:

$K=\sqrt{\frac{D_{h}}{2 R}} \frac{\rho U_{f} D_{h}}{\mu}=\sqrt{\frac{D_{h}}{2 R}} R e=\delta^{0.5} R e$

where

$D_{h}=2 a b /(a+b)$

$R e=\frac{\rho U_{f} D_{h}}{\mu}$

where $\mu$ is the fluid viscosity $(\mathrm{kg} / \mathrm{ms}), U_{f}$ is the mean fluid velocity $(\mathrm{m} / \mathrm{s}), D_{h}$ is the hydraulic diameter ( $\mathrm{a}$ and $\mathrm{b}$ are given as the microchannel cross section dimensions), $R$ is the flow path radius of the microchannel curvature $(m), \delta$ is the curvature ratio and $R e$ is the Reynolds number. As can be deduced from the given equation, the higher the fluid velocity is, the stronger the Dean vortices become. Furthermore, the intensity of the secondary flows also increases with the degree of curvature due to centrifugal effects. Thus, the proposed mixer having a curvature degree of $280^{\circ}$ is expected to be superior to its counterparts having a curvature degree of $180^{\circ}$ (J. J. Chen, Chen, and Shie 2011). The combination of a high curvature degree with alternating directions of Dean vortices due to repeating changes in the 
direction of pathway provides an enhanced mixing capability. At each inflection point, the Dean vortices are reconstructed as rotating in opposite directions with respect to their antecedent streamlines.

Another crucial dimensionless number, which needs to be taken into account for micromixing, is the Peclet number defined as $e=u D_{h} / D$, where $u$ is the characteristic velocity $(\mathrm{m} / \mathrm{s})$ and $D$ is the characteristic diffusion coefficient $\left(\mathrm{m}^{2} / \mathrm{s}\right)$. Pe is the ratio of mass transfer by convection to mass transfer by diffusion (J. J. Chen, Chen, and Shie 2011). Thus, for $P e<1$, the diffusion dominates over convection. On the other hand, forced convection generated by external forces or microchannel geometry dominates over diffusion for larger values (Marques and Fernandes 2011). Due to relatively high flow rates ranging from 100 to $3000 \mu \mathrm{l} / \mathrm{min}$ and large hydraulic diameters, the Peclet number (ranges from 7375 to 221260 ) and thus, the effect of chaotic advection on mixing is high.

The aim of this work is to offer effective curvilinear designs by scrutinizing the superiority of an asymmetrical micromixer over a symmetrical micromixer for achieving superior mixing. Proposed mixing is based on breaking the cyclic streamlines in curvilinear microchannels by width shifts so that the equilibrium state of inter-layer between the reagents is disrupted. Figure 1 displays the examined micromixers. 


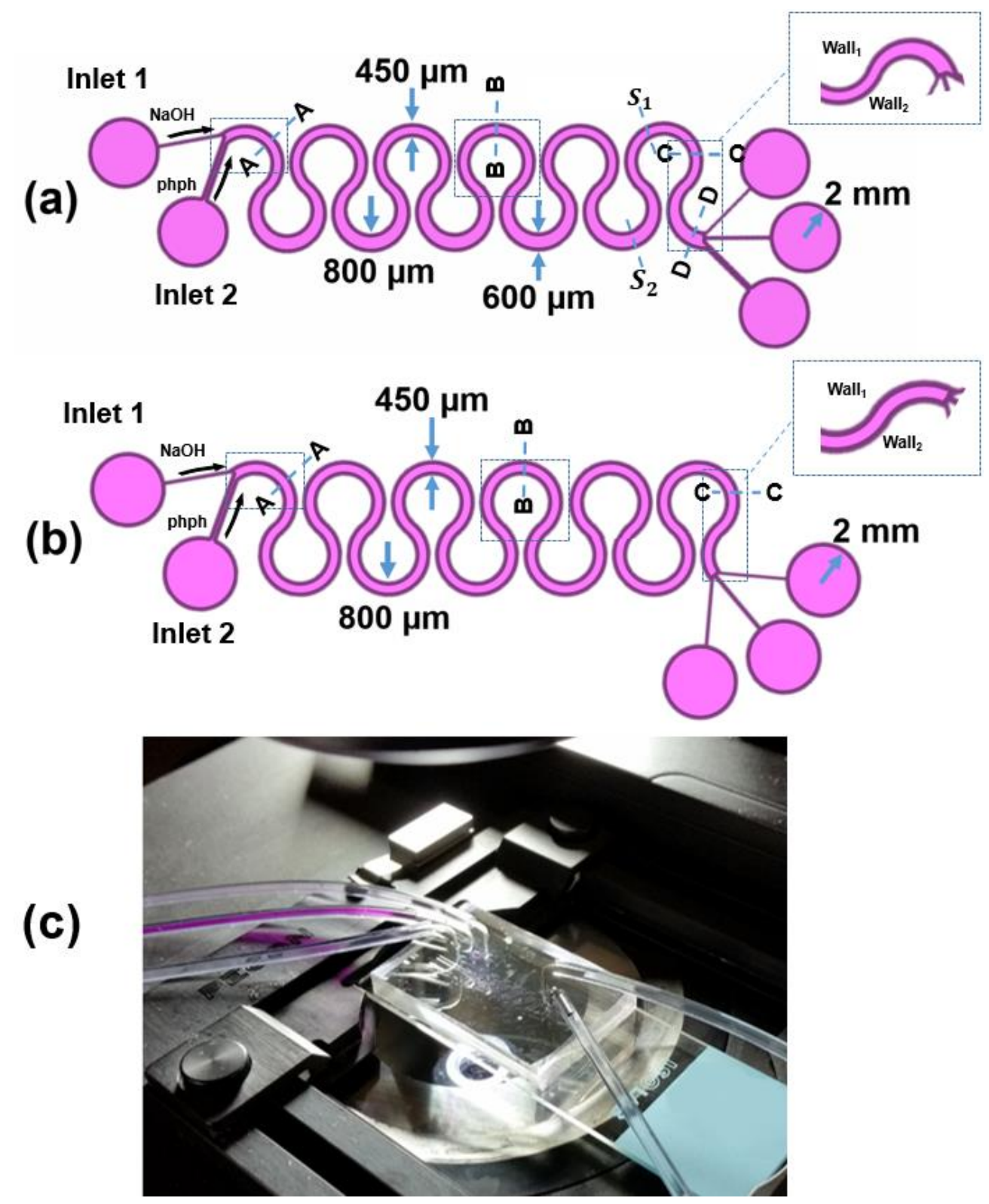

Figure 1: Designs of a) asymmetrical micromixer having a varying width ( $450 \mu \mathrm{m}$ to 600 $\mu \mathrm{m})$ and b) symmetrical micromixer having a width of $450 \mu \mathrm{m}$, which demonstrate the geometry of the microchannels, the mixing index evaluation lines indicated as A-A, B-B, $\mathrm{C}-\mathrm{C}$, and the velocity profile lines indicated as $S_{1}, S_{2}$ and D-D. The blue dashed rectangles show the locations, where the images were taken. c) The image of the experimental device, which shows the aqueous solutions of sodium hydroxide $(\mathrm{NaOH})$ and Phenolphthalein (phph) introduced to the inlets (on the right) while depicting the resulting fuchsia color after the mixing process (on the left). 


\subsection{Microchannel Fabrication}

\subsubsection{Fabrication Materials}

The PDMS (polydimethylsiloxane) microchannel preference emerged from its features, being highly compatible with this study, such as ease of fabrication, ease of visualization, indifference to chancing $\mathrm{pH}$ values and the cost efficiency. The end state of the PDMS microchannel is obtained through several fabrication steps, and the essential materials used in each individual step can be summarized as Su-8 3050 series for negative photoresist coating, acetate mask for UV lithography, $\mathrm{Su}-8$ developer for removal of excess material and PDMS with curing agent for molding the microchannel structure. The final step of the fabrication procedure is the $\mathrm{O} 2$ plasma bonding of PDMS microchannel to a glass substrate, which will be further explained in the following sections.

The physical properties of the preferred SU-8 negative photoresist provided by MicroChem corporation are given in Table 1. The SU-8 3050 negative photoresist was preferred for the purpose of this study since SU-8 3050 series can provide coating thicknesses up to $110 \mu \mathrm{m}$.

Table 1: The physical properties of SU-8 3050 provided by MicroChem corporation

\begin{tabular}{|c|c|}
\hline \multicolumn{2}{c}{ SU-8 3050 physical properties } \\
\hline Adhesion Strength - Silicon/Glass & $69 / 35 \mathrm{mPA}$ \\
\hline Thermal Stability & $300^{\circ} \mathrm{C}$ \\
\hline Thermal Conductivity & $0.2 \mathrm{~W} / \mathrm{mK}$ \\
\hline Coefficent of Thermal Expansion & $52 \mathrm{CTE} \mathrm{ppm}$ \\
\hline Tensile Strength & $73 \mathrm{Mpa}$ \\
\hline Elongation at break & $4.8(\varepsilon \mathrm{b} \%)$ \\
\hline Young's modulus & $2.0 \mathrm{Gpa}$ \\
Dielectric Constant at $1 \mathrm{GHz}$ & 3.28 \\
Bulk resistivity & $7.8 \times 10^{14}(\Omega \mathrm{cm})$ \\
\hline
\end{tabular}

The Acetate masks used in UV lithography was produced by CAD/Art Services, Inc. conformable to the specified designs. The 2-D designs of the symmetrical and asymmetrical microchannels can be seen in Figure 2. 

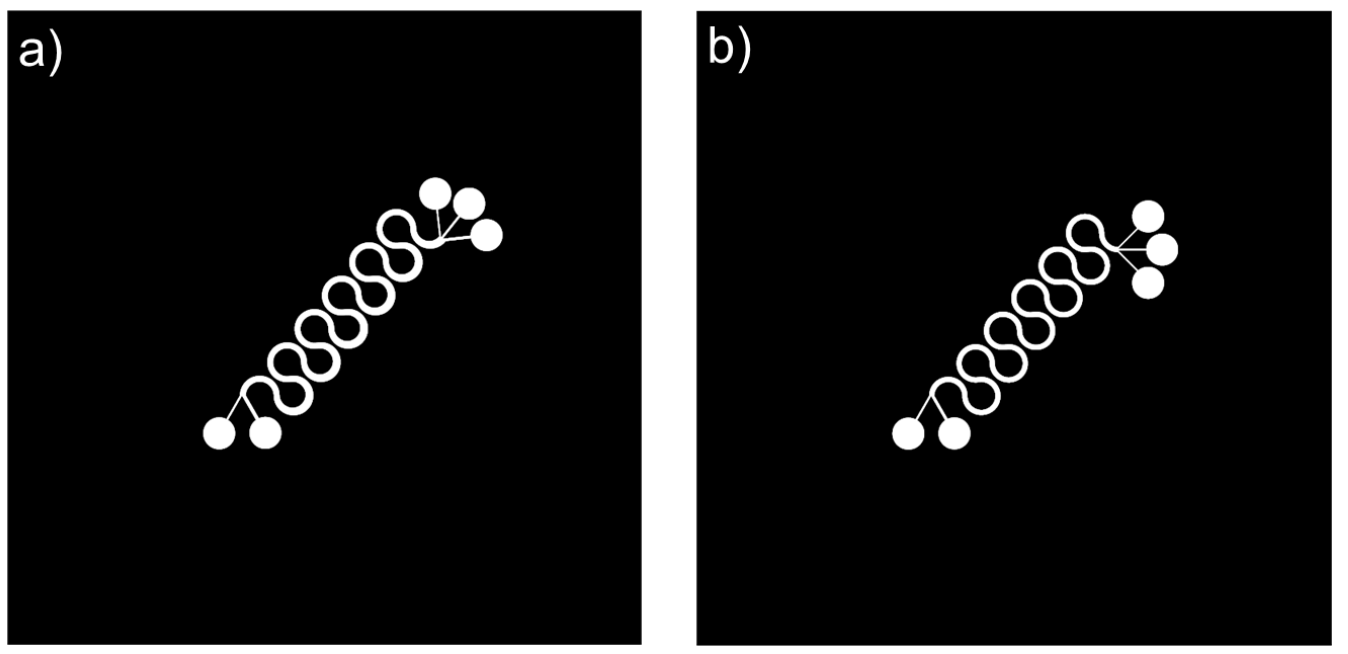

Figure 2: The 2D acetate mask designs of a) asymmetrical microchannel b) symmetrical microchannel

For the molding of the microchannels, the PDMS solution and the respective curing agent were acquired from Sigma Aldrich company under the name of Sylgard 184 silicone elastomer. The related PDMS material properties taken into consideration are given in Table 2.

Table 2: The material properties of PDMS provided by Dow corning corporation

\begin{tabular}{c|c}
\hline \multicolumn{2}{c}{ PDMS material properties } \\
\hline Color & Colorless \\
\hline Viscosity (Base) & $5175 \mathrm{cP}$ \\
\hline Viscosity (Mixed) & $3500 \mathrm{cP}$ \\
\hline Specific Gravity (Uncured) & 1.03 \\
\hline Specific Gravity (Cured) & 1.04 \\
\hline Mass density & $0.97 \mathrm{~kg} / \mathrm{m}^{3}$ \\
\hline Young's modulus & $360-870 \mathrm{KPa}$ \\
\hline Poisson ratio & 0.5 \\
\hline Tensile strength & $7.1 \mathrm{MPa}$ \\
\hline Specific heat & $1.46 \mathrm{~kJ} / \mathrm{kg} \mathrm{K}$ \\
\hline Thermal conductivity & $0.15 \mathrm{~W} / \mathrm{m} \mathrm{K}^{2}$ \\
\hline Index of refraction & 1.4 \\
\hline
\end{tabular}




\subsubsection{Fabrication Procedure}

Fabrication of the microchannels were conducted in an ISO class 2 cleanroom facility of Sabanci University Nanotechnology Centre (SUNUM). The procedure initiated with the negative photoresist coating of 3-inch single side polished wafers. Prior to the first step of the fabrication procedure, the wafers were cleaned and rinsed with isopropanol alcohol on the wet bench then left on a temperature controlled hot plate in order get rid of any residues or dust particle.

Due to the high viscosity of the negative photoresist, a computer controlled spin coater was used for the coating process. The use of such spin coater provides the efficacy of uniform height distribution throughout the wafer surface and decreases the possibility of bead formation at the wafer edges. The coating of the Su-8 3050 negative photoresist was conducted conformable to the parameters provided by the MicroChem company in order to obtain a thickness of $\sim 88 \mu \mathrm{m}$ (Figure 3 ).

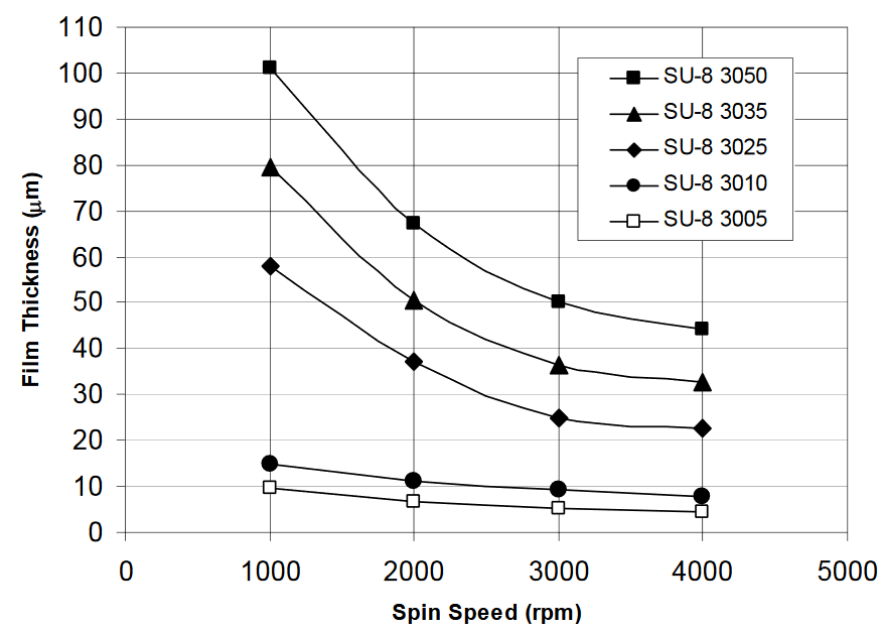

Figure 3: Film thickness vs spin speed chart of SU-8 3050 provided by MicroChem corporation

The introductory step of the spin coating was carefully centering the wafer and the wafer holder of the spin coater. The wafer holder was coupled with a vacuum mechanism that provides the immobilization of the wafer. Then pre-weighed $3 \mathrm{ml}$ negative photoresist was poured onto the center of the 3-inch wafer. The necessity of centering the wafer and the pouring spot is imperative for achieving a uniform propagation of the photoresist throughout 
the wafer. The spinning program of the computer controlled coater was divided into two parts and set to an initial $500 \mathrm{rpm}$ for 12 seconds then followed by $1800 \mathrm{rpm}$ for 30 seconds. The specified spin rates provided the desired thickness of $\sim 88 \mu \mathrm{m}$.

The second step of the spin coating was soft bake of the coated wafer so that the wafer was transferred onto a temperature controlled hot plate, where the soft bake temperature was set to $95^{\circ}$ and the wafer was kept on the hot plate for 40 minutes. The specifications taken into consideration for the soft bake and post exposure bake (PEB) were obtained from the SU-8 3050 data sheet given in Table 3.

Table 3: The process specifications of SU-8 3050 provided by MicroChem corporation

\begin{tabular}{c|c|c|c|c|c}
\hline \multicolumn{5}{c}{ Process specifications for SU-8 3050 } \\
\hline Thickness $(\mu \mathrm{m})$ & $\begin{array}{c}\text { Soft Bake Duration } \\
(\mathrm{min})\end{array}$ & $\begin{array}{c}\text { Exposure Energy } \\
(\mathrm{MJ} / \mathrm{cm} 2)\end{array}$ & $\begin{array}{c}\text { PEB Time at } 65^{\circ} \mathrm{C} \\
(\mathrm{min})\end{array}$ & $\begin{array}{c}\text { PEB Time at 95 } \\
(\mathrm{min})\end{array}$ & $\begin{array}{c}\text { Development Duration } \\
(\mathrm{min})\end{array}$ \\
\hline $4-10$ & $2-3$ & $100-200$ & 1 & $1-2$ & $1-3$ \\
$8-15$ & $5-10$ & $125-200$ & 1 & $2-4$ & $4-6$ \\
\hline $20-50$ & $10-15$ & $150-250$ & 1 & $3-5$ & $5-8$ \\
\hline $30-80$ & $10-30$ & $150-250$ & 1 & $3-12$ \\
\hline
\end{tabular}

The UV light exposure process began with the end of soft bake process. The coated wafer was carefully mounted on the Midas/MDA-60MS Mask Aligner wafer holder and then the aforementioned acetate mask was centered onto the respective mask holder. After the alignment of the wafer and the acetate mask, the UV light exposure was initiated, where the duration of the $23 \mathrm{MJ} / \mathrm{s} . \mathrm{cm}^{2}$ energy input was set to 13 seconds. At the end of this process, it was necessary to have a delay before the placement of the coated wafer onto the hot plate in order to relieve $\mathrm{N}_{2}$ from the coated thick photoresist. Otherwise, the $\mathrm{N}_{2}$ inside the thick coated photoresists might have expanded and thus induced more undesired mechanical stress.

The subsequent step of the fabrication process was the post exposure bake thus the exposed wafer was placed onto the hot plate, and the process was conducted conformable to the exact values previously given in Table 3. The importance of post exposure bake arises from the fact that while reducing the mechanical stress caused by nitrogen, it also improves 
the thermally induced diffusion of carboxylic acid inside the photoresist, thereby providing a better spatial resolution and aspect ratio. For the initial post exposure baking, the wafer was rested on a $65^{\circ} \mathrm{C}$ hot plate for a duration of 1 minute followed by the second step of the post exposure bake, where the coated wafer was rested on a $95{ }^{\circ} \mathrm{C}$ hot plate for a duration of 5 minutes.

Following this process, the coated wafer was placed in a petri dish containing the respective developer in order to get rid of the excess unexposed photoresist and to obtain the desired pattern, which will be used as the mold. Afterwards, the specified petri dish was transferred into an ultrasonic bath for the execution of 13-minute vibration. After the desired pattern was obtained, the patterned wafer was rinsed and dried with isopropanol alcohol and $\mathrm{N}_{2}$ respectively. Since the patterned wafer was used as a mold for the purpose of this study, a final hard baking process was implemented, where the patterned wafer was rested on a hot plate which has a temperature of $150^{\circ} \mathrm{C}$ for 120 minutes for further hardening.

The penultimate step was the PDMS molding of the finalized wafer with the desired pattern. For this process, the inside of a commercially used $100 \mathrm{~mm}$ petri dish was covered with a thin aluminum foil. Then, two sided tapes were affixed between the aluminum foil and the patterned wafer to achieve stability. The preparation of the PDMS solution, which was to be poured into the petri dish, was conducted on a weigh tray by introducing both liquid PDMS and the respective curing agent with a ratio of 10:1. The mixture was manually well stirred with the use of a glass stick for mixing purposes. After the mixture was complete, the solution was ready to be poured onto the center of the petri dish containing the patterned wafer. The bubble formation arising from the mixture of PDMS solution and the curing agent was inevitable; however, to overcome this problem the petri dish was put in an oven with vacuum feature for 40 minutes thus the PDMS solution was degassed up to the point, where no bubble was observed in a visual inspection. Then, for the hardening of the PDMS, the vacuum oven was set to a temperature of $75^{\circ} \mathrm{C}$, and the petri dish was kept inside the chamber for 14 hours.

The latter step of the fabrication process was to conscientiously separate the solid PDMS from the patterned wafer, which could be reused for the production of more identical PDMS microchannels. Finally, the solid PDMS section containing the microchannel was cut into a rectangular shape compatible with the commercially available glass substrates by using 
a scalpel. For better comprehension, the essential steps of the PDMS microchannel fabrication process is illustrated in Figure 4.

a)

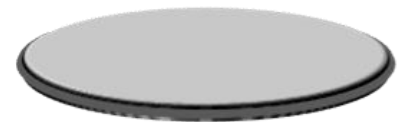

Single-sided wafer

b)

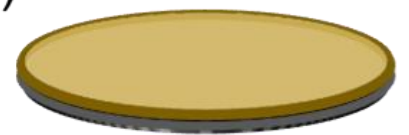

Photoresist coat

C)
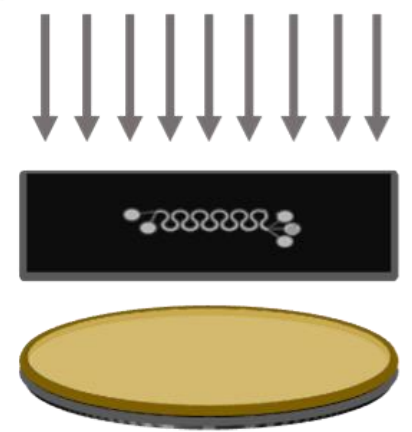

UV light exposure d)

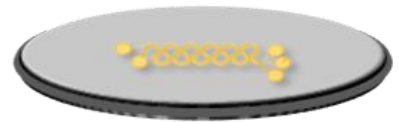

Patterned wafer

e)

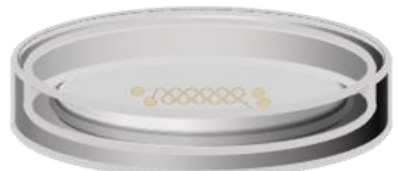

PDMS mold

f)

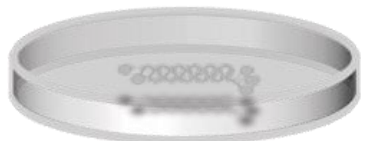

Patterned solid PDMS

g)

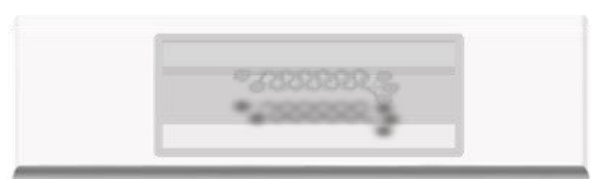

$\mathrm{O}_{2}$ plasma bonded microchannel

Figure 4: Illustration of the essential steps in PDMS microchannel fabrication process

\subsubsection{O2 Plasma Bonding}

The $\mathrm{O}_{2}$ plasma bonding process requires highly purified chamber ambient and dirt free surfaces in order to perform effectively. Thus, at the beginning of the process, the chamber of the Harrick High Power Plasma Cleaner was wiped off and the both PDMS, and glass substrate was cleaned with isopropanol alcohol. Since the surface modification of the PDMS and glass substrate caused by highly energetic oxygen ions might be disrupted by the undesired particles in the chamber and on the substrates, conducting the pre-cleaning process was crucial prior to the bonding process.

The process proceeded with the placement of the PDMS and the glass substrate into the chamber, where the microchannel pattern side of the PDMS was placed facing up. The 
chamber was then vacuumed with the use of a dry oxygen service pump, and the device was set to a high radio frequency setting, which provided a power of $45 \mathrm{~W}$. Afterwards, the PDMS and the glass substrate were subjected to $\mathrm{O}_{2}$ plasma for 60 seconds, where the dose was controlled to be $10 \mathrm{ml} / \mathrm{min}$ during the entire process. At the end, the chamber was refilled with air, and the coupling of PDMS and glass substrate was quickly performed with precise alignment.

The final step of the $\mathrm{O}_{2}$ plasma bonding process was placing the combined substrate onto a $75^{\circ} \mathrm{C}$ hot plate for 20 minutes for further improving the adhesion between the interfaces.

\subsection{Mixing Materials and Experimental Setup}

\subsubsection{Mixing Materials}

In order to analyze the mixing efficiency of the micromixers, a $\mathrm{pH}$ indicator (Phenolphthalein), which demonstrates a transition from transparent to fuchsia color with a change from acidic conditions to the basic conditions $(\mathrm{pH}>8.2)$, was preferred. In addition to Phenolphthalein, a basic solution of $\mathrm{NaOH}(\mathrm{pH}-13)$ was chosen since it has the feature of being transparent. The transition from transparency to colorful pattern is essential for studies visualized by monochromatic cameras. Once two solutions are introduced to channel inlets, the mixture acquires a fuchsia color, while the regions, where no mixing occurs, remain transparent.

\subsubsection{Equipment and Programs}

For each experiment, two $60 \mathrm{~mL}$ plastic syringes were loaded with $\mathrm{NaOH}$ and Phenolphthalein solutions and were injected into the microfluidics device (micromixer) at flow rates ranging from 100 to $3000 \mu \mathrm{l} / \mathrm{min}$ using a syringe pump (KD Scientific Legato 200). The connections between the microfluidics device and syringe/collection tubes were accomplished using TYGON tubings (IDEX Corp., IL) (internal diameter: $250 \mu \mathrm{m}$ length:150 mm) and corresponding fittings (IDEX Corp., IL). The device was mounted on an inverted phase contrast microscope (Leica DMIL LED) equipped with a high speed 
camera (Phantom v310). The images were obtained using high speed camera videos and were recorded at a specified region near the channel outlet with a frame rate of $300 \mathrm{fps}$ and an exposure time of $200 \mu \mathrm{s}$.

\subsubsection{Sample Preparation}

The sample preparation was initiated with the cleaning and rinsing of the PDMS microchannels and the TYGON tubing with Ethanol, then followed by injection of a $70 \%$ Ethanol - 30\% DI water mixture into the microchannel to prevent inconveniences, which might be caused by dirt or dust. However, it should be noted that the deposition of small dust particles from the air in the process of experimentation was non-preventable. Subsequent to the microchannel and tubing cleaning procedure, $300 \mathrm{~mol} / \mathrm{m}^{3}$ Phenolphthalein and $10 \mathrm{~mol} / \mathrm{m}^{3}$ $\mathrm{NaOH}$ were dissolved in 70\% Ethanol - 30\% DI water mixture inside a commercially used falcon tube. The homogeneity of the solutions was obtained by putting the falcon tubes in an ultrasonic bath as vibration mechanism. Considering the purpose of this study, the reaction time for Phenolphthalein and sodium hydroxide was negligible. Then, each solution was transferred into respective pre-cleaned $60 \mathrm{~mL}$ plastic syringes mounted on the syringe pump. Consequently, the tubing connections between the microchannel and the syringes were integrated, where the microchannel was already placed onto the visualization system as seen in Figure 1c.

\subsection{Mixing Rate Analysis Procedure}

Raw images, which were obtained using the aforementioned high speed camera, were converted to 8-bit grayscale images, which gave 256 different intensities of gray, in the ImageJ software. A plotting line profile module shown as the $\mathrm{C}-\mathrm{C}$ line (Figure 1) was used to achieve the pixel intensity values, pixel by pixel, across the channel width near the outlet region. A normalization process is required since each image possesses a different intensity level ranging from 0 to 255 . The gray values are normalized to be 0 for the minimum intensity value (unmixed region) and 1 for the maximum intensity value (completely mixed region). The mixing index was then calculated in parallel lines with the literature (Cha et al. 2006) as follows: 


$$
\begin{aligned}
& I_{N i}=\frac{I_{i}-I_{0}}{I_{1}-I_{0}} \\
& M I=1-\sqrt{\frac{1}{N}} \sum_{i=1}^{N}\left(1-I_{N i}\right)^{2}
\end{aligned}
$$

where $I_{N i}$ is defined as the normalized pixel intensity, $I_{i}$ is the related pixel intensity, $I_{0}$ is the intensity at the unmixed zones, $I_{1}$ is the intensity at the fully mixed zones and $N$ is the number of pixels (Cha et al. 2006). I0 was calculated using the pixel intensity average of two images obtained from two separate experiments. In the first experiment, only Phenolphthalein was passed, while $\mathrm{NaOH}$ (at the exact molarity as in the experiments) was introduced into the microchannel in the second experiment. The pixel intensity average was always obtained from the same line, where the mixing index was evaluated (Figure 1). The relative $I_{0}$ was used to keep the light intensity the same for each experiment. Before each experiment, this procedure was followed to eliminate any difference, which might occur among the experiments due intensity fluctuations obtained from the high speed camera.

$I_{1}$ was found for each experiment by introducing a pre-mixed solution of Phenolphthalein and $\mathrm{NaOH}$ (at the exact molarity as in the experiments) into the microchannel. The pixel intensity average was again obtained from the same line, where the mixing index was evaluated (Figure 1). The same procedure was followed before each experiment to obtain the same light intensity from the microscope so that the differences, which might occur among the experiments due intensity fluctuations obtained from the high speed camera, could be eliminated.

In order to ensure the reliability of the mixing analysis, prior to mixing data collection, the procedure included the visual examination of occurrence of any dirt along the pixel intensity lines, the inquisition of pixel histogram for any abnormal decline in the pixel intensity and finally the placement of the pixel intensity line. First step of the placing procedure allowed to move the PDMS microchannel on the microscope if any noticeable sized dirt was inspected along the line of relative pixel intensity line. The second step resulted in eliminating the smaller pieces of dirt missed in the visual examination. Abnormal decreases in pixel intensity were caught in this procedure. If there were any relatively smaller 
pieces of dirt along the line of relative pixel intensity line, the final step of the procedure began, where the pixel line was carefully placed next to original pixel intensity line with a tolerance of at most $15 \mu \mathrm{m}$, which corresponded to a shift of 5 pixels further from the original intensity line.

\subsection{CFD Model Formulation}

Fluid flow in the micromixers was modeled using the Comsol Multiphysics 5.2 software (COMSOL Inc., Burlington, MA) for a qualitative mixing efficiency analysis. The dimensions used in the 3D model was kept as the same as the experimental configuration as shown in Figure 1. The mesh of the COMSOL model (3D) was generated by means of physics-controlled extremely fine mesh elements of 6,180,736 with different mesh element size parameters. The optimum mesh element structure can be seen in Figure 5.

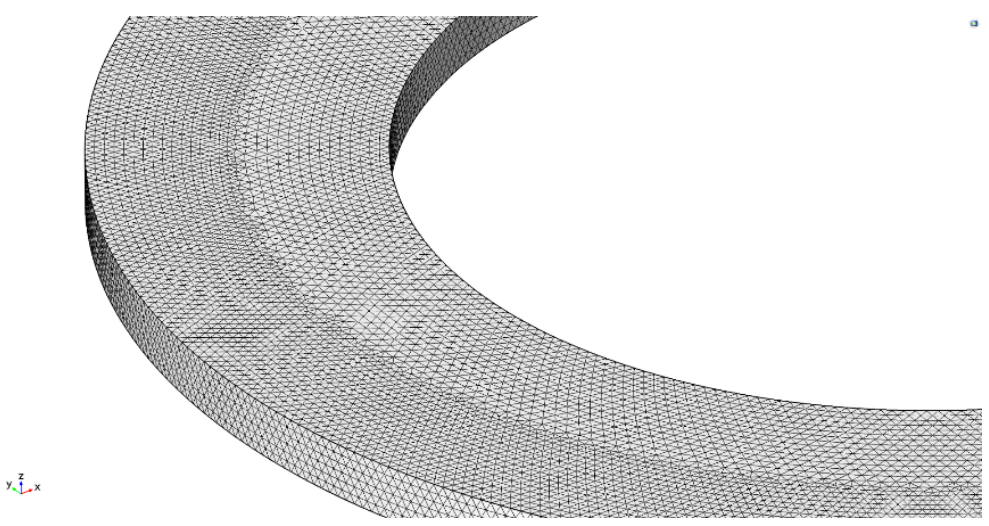

Figure 5: The optimum mesh element structure generated for the computational study

GMERS solver was chosen among other default solvers with 6,915,321 degrees of freedom. The complete mesh consists of 7,283,598 domain elements, 559,230 boundary elements, and 14,589 edge elements. The simulations were performed in a workstation with an ASUS G752VT Intel Core i7-6700HQ 2.6 GHz processor with 24 GB RAM. The server's operating system is Windows 7 Professional 64-bit edition.

The simulations were performed by solving the Navier-Stokes equations via the Laminar Flow Model module and assuming the fluid flow to be single phase, steady state and incompressible. Boundary conditions of the no-slip (zero velocity condition) on the walls 
and zero pressure at the outlet were imposed. The governing Navier-Stokes equations for an incompressible and steady state laminar flow are expressed as:

$\rho(u . \nabla) u=\nabla \cdot\left[-p I+\mu\left(\nabla u+(\nabla u)^{T}\right)\right]$

where $u$ is the flow velocity vector, $\rho$ is the fluid density, $\mu$ is the fluid viscosity and $p$ is the pressure. The effect of the presence of Phenolphthalein and $\mathrm{NaOH}$ on fluid properties is neglected, and therefore fluid velocity and viscosity values were chosen for $70 \%$ ethanol and $30 \%$ DI water mixture at room temperature (J. J. Chen, Chen, and Shie 2011). Various flow rates between 100 and $3000 \mu 1 /$ min corresponding to Reynolds numbers between 2 and 59 were imposed at the inlet of the microchannels. Consequently, from the steady-state solution of laminar flows, mean flow velocity maxima positions along the channel and Dean flow velocity profiles at the particular locations were obtained. Simulation results give additional insights to mixing efficiencies and help explaining the results. 
CHAPTER 3

RESULTS AND DISCUSSION 


\subsection{Experimental Results}

In Poiseuille flows, the velocity profile increases from zero near the channel walls to a maximum at the centerline of the channel cross-section. For microchannels with curvilinearity, due to this velocity profile, fluid particles closer to the walls attain relatively smaller inertia compared to those at the centerline thereby creating a movement towards the outer wall, which results from this radial pressure gradient when encountering a curve. This radial movement is also called secondary flow. Microchannel designs with curvilinearity redirect the downstream fluid flow at each turn, and this effect comes into sight, when the velocity maxima at the centerline along the pathway tend to shift towards the outer wall at each bend, which causes reorienting secondary flows including Dean vortices. Owing to the geometry of the micromixers presented in this study, the velocity profile including the Dean vortices after each turn is exploited for effective mixing.

The effect of width asymmetry on mixing efficiency is revealed for both micromixers at Reynolds numbers based on the cross-sectional area at the entrance having a width of 450 $\mu \mathrm{m}$. While the corresponding Reynolds number $\left(R e_{1}\right)$ ranges from 2 to 59 , the ratio of the Reynolds number at the wider region of the asymmetrical microchannel $\left(R e_{2}\right)$ to the one at the entrance is found to be 0.78 , which corresponds to a Reynolds number $\left(R e_{2}\right)$ range from 1.53 to 46 . The corresponding Dean number with respect to the entrance ranges from 0.84 to 25.2 .

In Figure 6, high speed camera images of the flow near the entrance can be seen at different Dean numbers. The images at Dean numbers, where a significant change in diffusion layer between the reagents occurs, are presented. 
(a)

(b)

(c)

(i)

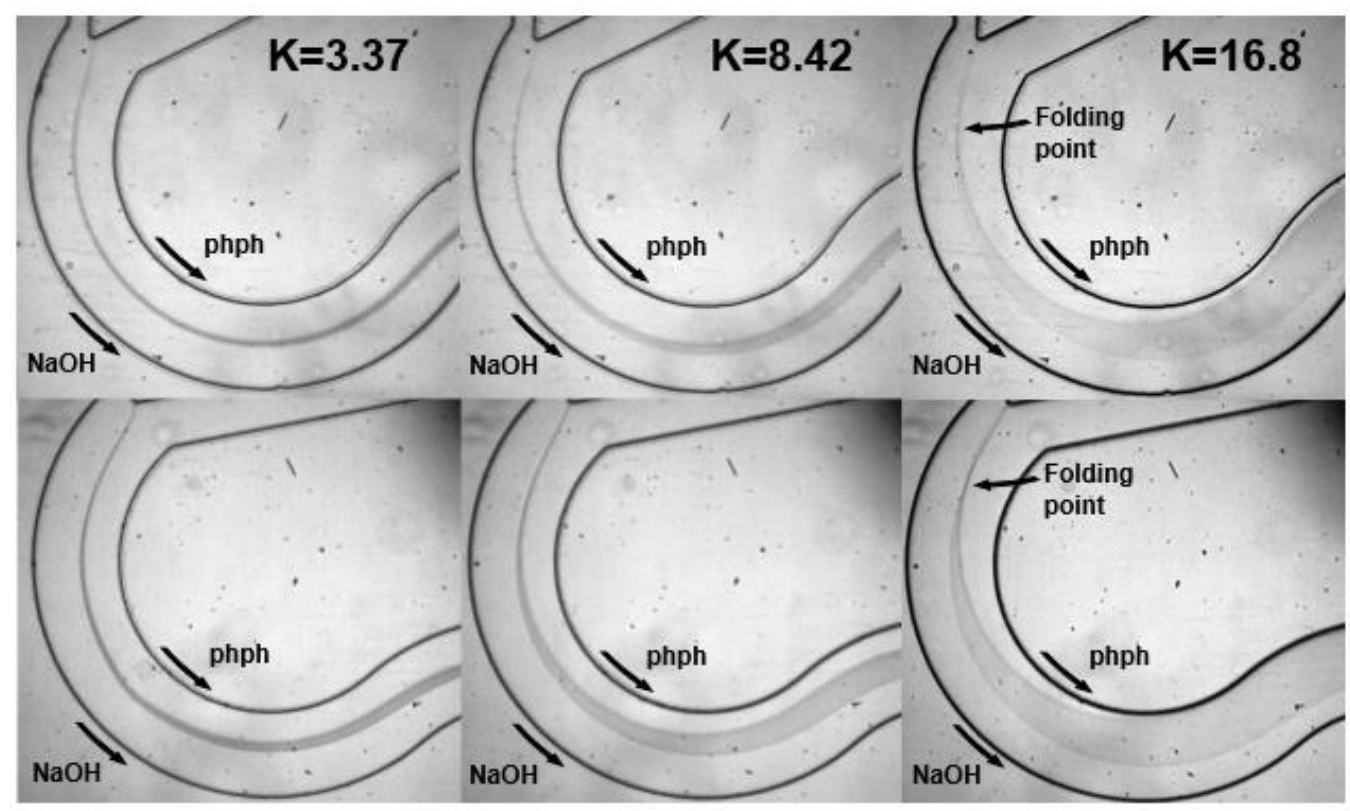

(d)

(e)

(i)

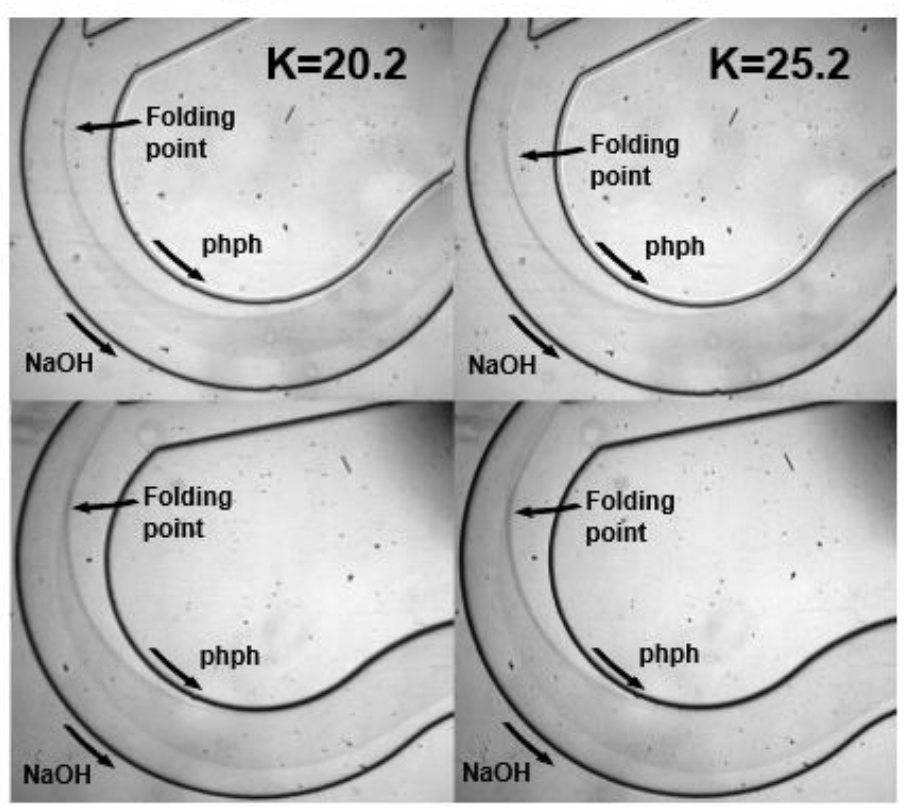

Figure 6: Mixing images of Phenolphthalein and $\mathrm{NaOH}$ aqueous solutions in: i) asymmetrical and ii) symmetrical micromixers at the given Dean numbers. The visualization was performed at the entrance region, and the corresponding flow rates are a) $400 \mu \mathrm{l} / \mathrm{min}$, b) $1000 \mu \mathrm{l} / \mathrm{min}$, c) $2000 \mu \mathrm{l} / \mathrm{min}$, d) $2400 \mu \mathrm{l} / \mathrm{min}$, e) $3000 \mu \mathrm{l} / \mathrm{min}$. 
As seen in Figure 6a, the curvilinearity (thus the effect of the secondary flows) at $\mathrm{K}=3.37$ is insufficient to spread the diffusion layer for both asymmetrical and symmetrical microchannels near the entrance. Taking the entrance into consideration, for the flow rate below $400 \mu \mathrm{l} / \mathrm{min}$, the velocity gradient between the center of the streamline and the regions near the walls is not big enough to form Dean vortices. The effect of Dean vortices near the entrance becomes more significant at $K=8.42$ (Figure $6 \mathrm{~b}$ ), since the diffusion layer between the reagents begins to spread towards the side of the Phenolphthalein. This shift towards the Phenolphthalein side may result from the difference in molarities. The reason for the shift in the diffusion layer towards the Phenolphthalein side instead of being at the centerline arises from this difference, which is a result of the dependence of diffusion on concentration difference. At $\mathrm{K}=16.8$ (Figure 6c), where the flow rate is $2000 \mu \mathrm{l} / \mathrm{min}$, the diffusion layer begins folding on itself in both microchannels, as the counter-rotating vortices push the center of the layer from the Phenolphthalein side. The trend is opposite near the upper and bottom walls. This folding motion leads to a point, where secondary flows begin to disperse the diffusion layer. The width of the diffusion layer rapidly increases beyond the folding point at high Dean numbers, ranging from $\mathrm{K}=16.8$ to $\mathrm{K}=25.2$ (Figures 6c-e). The high curvature angle of the proposed microchannel causes earlier secondary flow formation thereby promoting a better mixing compared to that in curvilinear microchannels with lower curvature angles. For both cases, the diffusion layer widens towards the Phenolphthalein side as a result of the difference in molarity between the solutions of $\mathrm{NaOH}$ and Phenolphthalein.

Figure 7 presents the images acquired at the sixth turn of the microchannel, which is also highlighted as a blue dashed rectangle in Figure 1. This figure consists of the images taken at the same Dean numbers as in Figure 6. 
(a)

(b)

(c)

(i)

(ii)

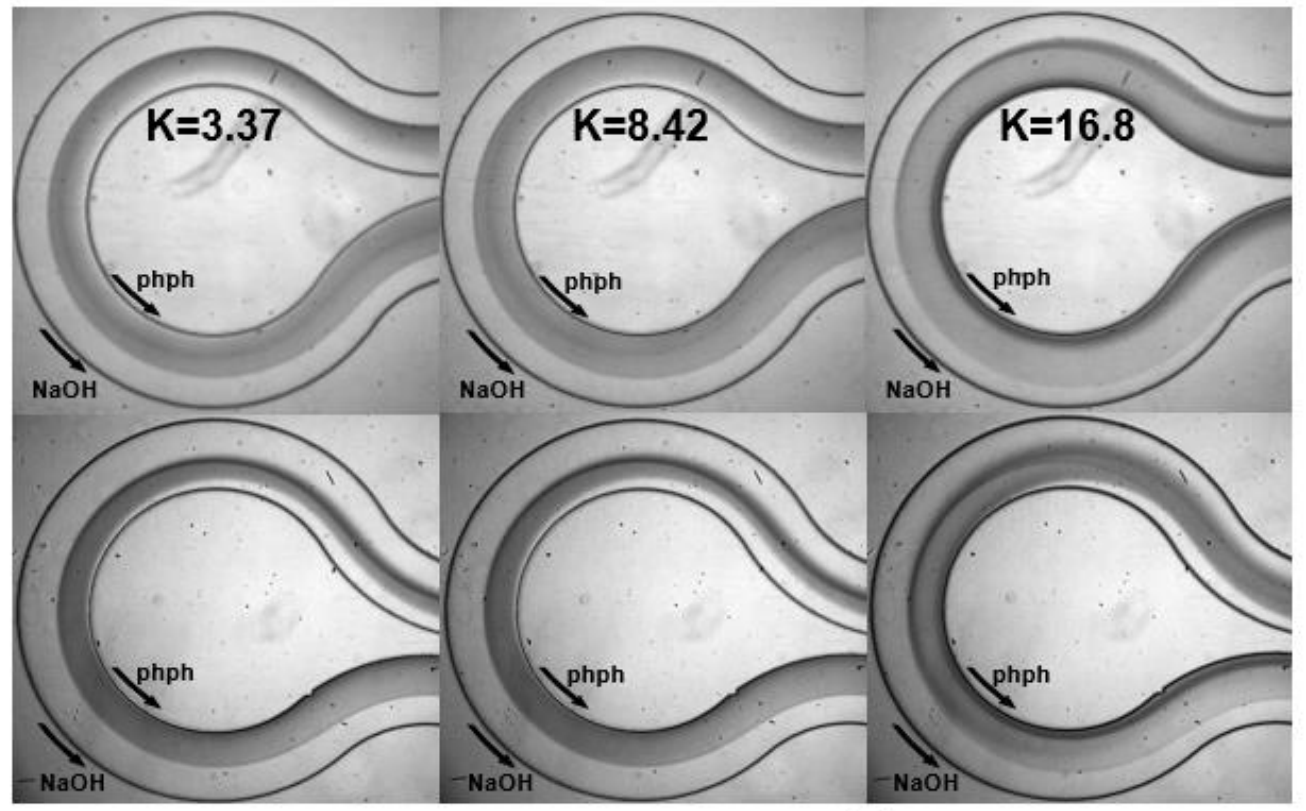

(d)

(e)

(i)

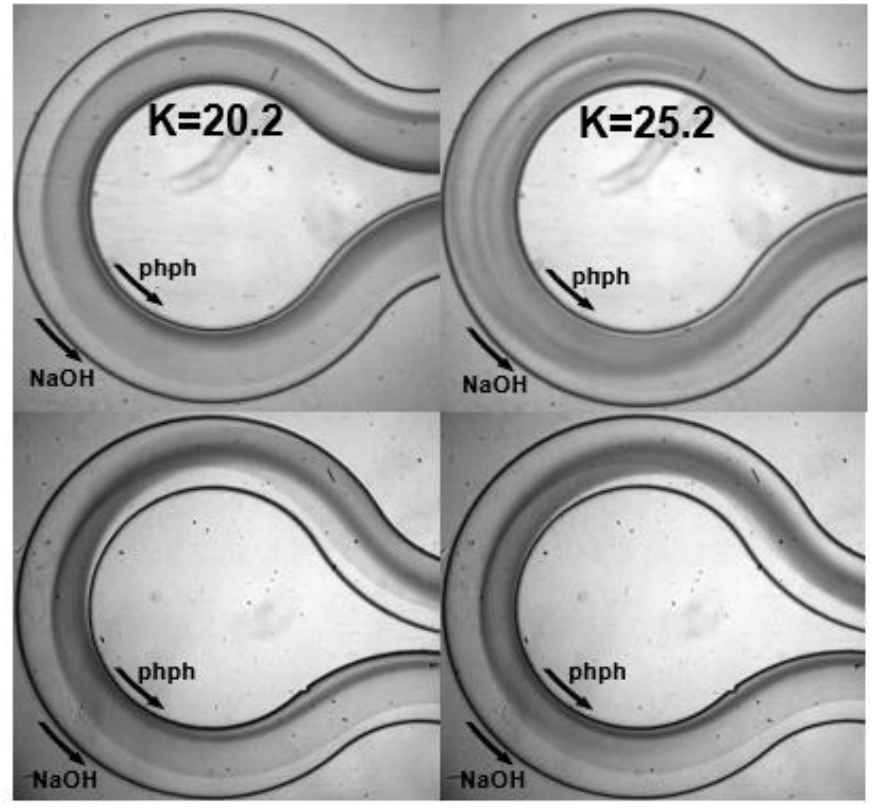

Figure 7: Mixing images of Phenolphthalein and $\mathrm{NaOH}$ aqueous solutions in: i) asymmetrical and ii) symmetrical micromixers at the given Dean numbers. The visualization was performed at the sixth turn of the microchannel, and the corresponding flow rates are a) $400 \mu 1 / \mathrm{min}$, b) $1000 \mu \mathrm{l} / \mathrm{min}$, c) $2000 \mu \mathrm{l} / \mathrm{min}$, d) $2400 \mu \mathrm{l} / \mathrm{min}$, e) 3000 $\mu 1 /$ min. 
As seen in Figure 7a, both asymmetrical and symmetrical microchannels have a diffusion layer with a similar width at $\mathrm{K}=3.37$. However, the asymmetrical one has a relatively lighter diffusion layer due to the stretching and contracting motion of Dean vortices due to the asymmetrical width. Between $\mathrm{K}=8.42$ and $\mathrm{K}=16.8$ (Figures $7 \mathrm{~b}-\mathrm{c}$ ), the same trend continues, and the diffusion proceeds towards the Phenolphthalein side in both microchannels. In Figure 7d and Figure 7e, the effect of strong secondary flows can be observed from the voids inside the diffusion layer of both microchannels, which is an indicator of the rotational streamlines as a consequence of chaotic advection. Figures $8 \mathrm{a}$ and Figure $8 \mathrm{~b}$ demonstrates the comparison of mixing performances of the microchannels near the entrance and sixth turn.

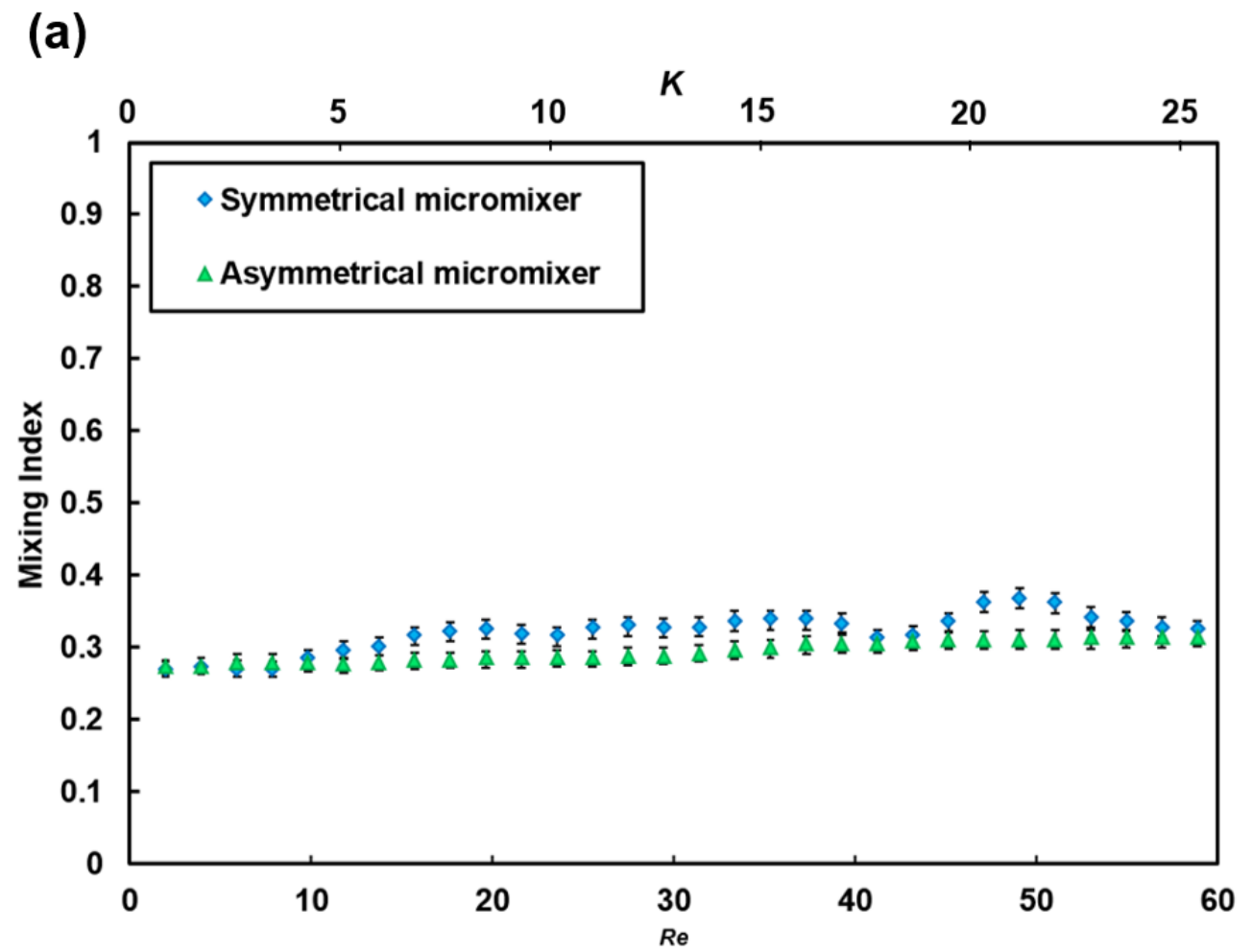




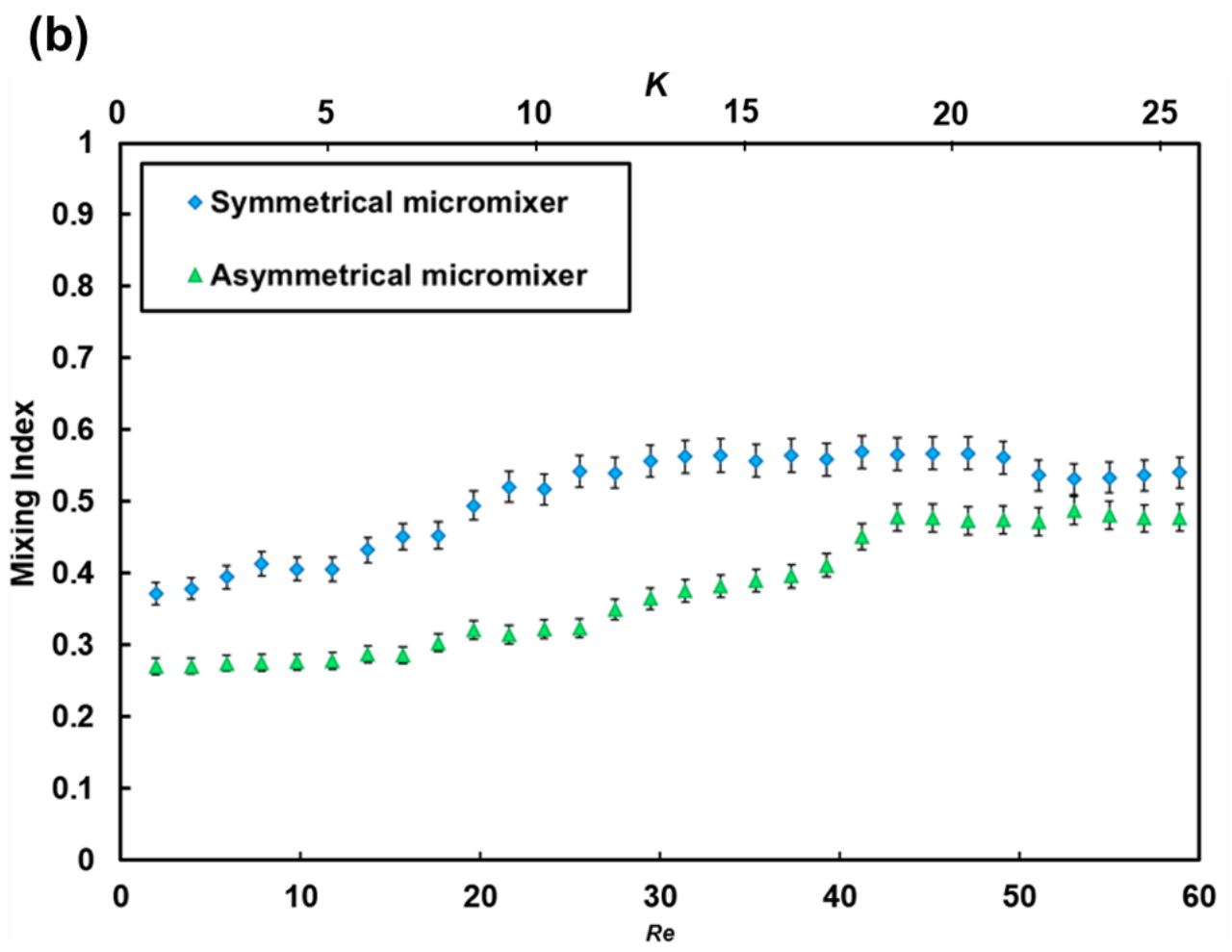

Figure 8: Mixing index as a function of Reynolds number (bottom x-axis) and Dean number (top x-axis) for symmetrical and asymmetrical micromixers at a) entrance b) sixth turn

Figure 8a shows that the mixing performances of the asymmetrical and symmetrical microchannels are very close to each other because of the negligible effect of curvilinearity near the entrance. However, the symmetrical microchannel is slightly superior to the asymmetrical one due to the widening wall at the next turn upstream of the asymmetrical microchannel. From the images provided in Figure 8b, it can be seen that at the sixth turn of both microchannels, the symmetrical microchannel outperforms the asymmetrical one. The inferior mixing performance of the asymmetrical microchannel stems from the relatively weaker Dean vortices at its wider regions. The asymmetrical microchannel benefits from both the secondary flows that form due to curvilinearity and the stretching and contracting motion of the two counter-rotating Dean vortices. In wider regions, the rotation of the vortices becomes weaker, whereas the asymmetry induces the motion of stretching and contracting thereby contributing to mixing. Figures $8 \mathrm{a}-\mathrm{b}$ indicate that the effect of the rotation 
of regular vortices inside the symmetrical microchannel is superior to the combined effect of the rotation of stretching and contracting vortices up to the sixth turn along the microchannels for all the Dean numbers $(0.84<\mathrm{K}<25.2)$.

Near the outlet region, the experimental data was divided into two parts based on the critical Dean number $K=16.8$ according to the flow trends. Above this number, the asymmetrical micromixer is more favorable for mixing compared to the equivalent symmetrical one. Figure 9 provides the visual data of both of the micromixers for $K$ values between 0.84 and 16 .

(a)

(b)

(c)

(i)

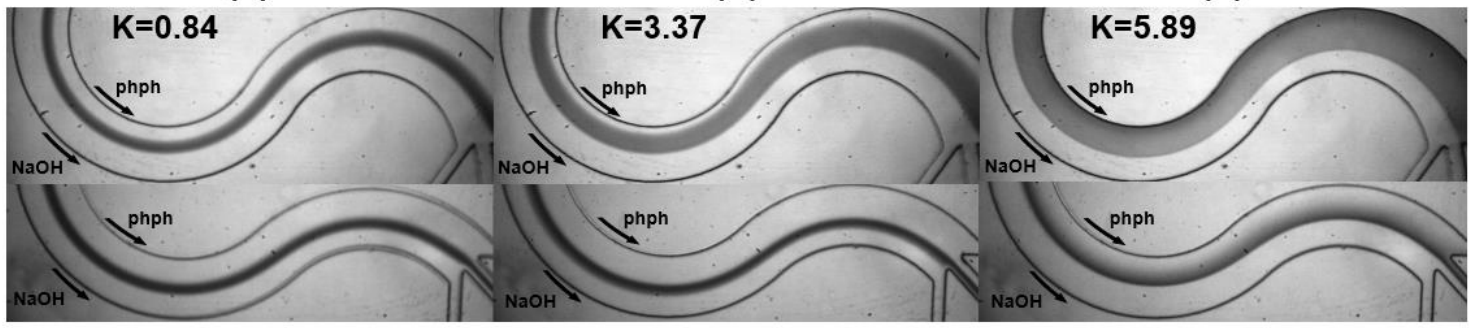

(d)

(e)

(i)

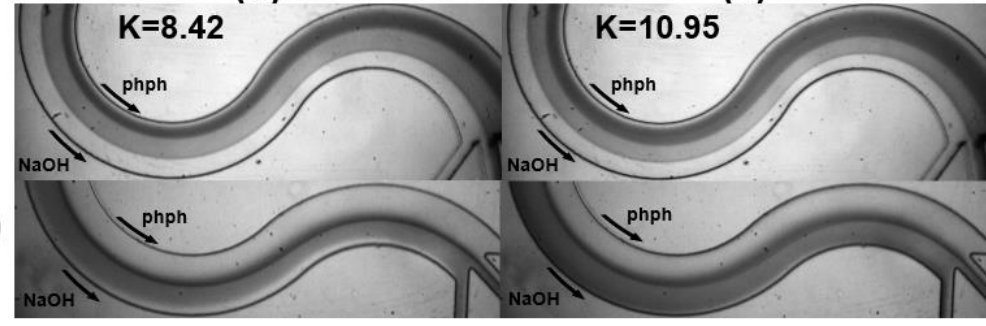

(f)

(g)

(i)

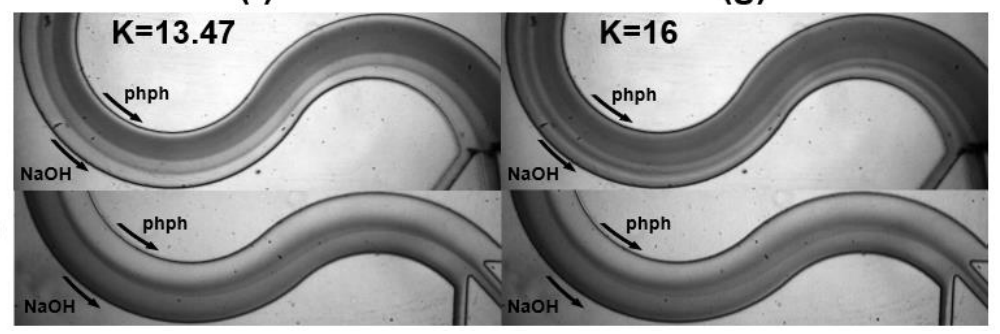

Figure 9: Visual mixing data of Phenolphthalein and $\mathrm{NaOH}$ aqueous solutions in: i) asymmetrical and ii) symmetrical micromixers for the given Dean numbers below the specified critical value $(\mathrm{K}<16.8)$. The visualization was performed at the outlet region where corresponding flow rates are a) $100 \mu \mathrm{l} / \mathrm{min}$, b) $400 \mu \mathrm{l} / \mathrm{min}$, c) $700 \mu \mathrm{l} / \mathrm{min}$, d) 1000 $\mu \mathrm{l} / \mathrm{min}$, e) $1300 \mu \mathrm{l} / \mathrm{min}$, f) $1600 \mu \mathrm{l} / \mathrm{min}$ and g) $1900 \mu \mathrm{l} / \mathrm{min}$ 
For Dean numbers less than 16.8 , it is difficult to reach a precise conclusion about whether the asymmetrical microchannel is superior when solely scrutinizing the visual data. Even though the asymmetrical microchannel stands out at first glance due to having a wider inter-diffusion area for almost every Dean number below the specified threshold, the symmetrical microchannel has a relatively darker inter-diffusion area. This variance in width and darkness occurs because the Dean vortices inside the asymmetrical microchannel lead to a stretching and contracting motion, which spreads the mixed layer thereby making it appear less dark. As it can be seen in Figure 9a, where the flow rate is $100 \mu 1 / \mathrm{min}$, the Reynolds and Dean numbers are comparably very small so that the effect of the Dean vortices inside the micromixers is negligible, which results in a similar thin diffusion layer near the centerline.

The Peclet number at this flow rate $(P e=7375)$ acts as a supporting parameter since the mixing is expected to be dominated by diffusion rather than chaotic advection for $P e<$ 10000 (Nguyen and Wu 2005), thereby leading to just a nuance of relatively wider mixture layer, while the symmetrical one exhibits a relatively darker mixture layer indicating a better performance. At low Dean numbers, the absence of high velocity gradient in the fluid particles in the Poiseuille velocity profile results in a lower fluid inertial effect thereby reducing the contribution of the Dean vortices to mixing up to a Dean number of $K=3.37$ (Figure 9b). In Figure 9b, it can be observed that the asymmetrical micromixer has a widened mixture layer, and the symmetrical one also displays a tendency of widening with a darker mixture layer. Both observations are a result of waxing Dean vortices since the Peclet number at this flow rate $(P e=29501)$ reflects the domination of chaotic advection. In addition, for the asymmetrical micromixer it can be seen that the diffusion layer is widening towards the Phenolphthalein side at this Dean number, which may result from the effect of molarity difference between the reagents. In Figure 9c, the trend of widening of the mixed layer prevails.

At $K=8.42$ (Figure 9d), the effect of stretching and contracting motion of the Dean vortices inside the asymmetrical microchannel becomes more conspicuous since the width of the mixed layer increases beyond the inflection point near the outlet where the widening of the microchannel from $450 \mu \mathrm{m}$ to $600 \mu \mathrm{m}$ occurs. The symmetrical micromixer in Figure $9 \mathrm{~d}$ also begins to demonstrate a trend of widening diffusion layer towards the $\mathrm{NaOH}$ side of 
the microchannel. At $K=10.95, K=13.47$ and $K=16$ (Figures 9e-g), the propagation of the mixed region from one wall to another continues for both microchannels, as the effect of the Dean vortices become more and more dominant with flow rate. However, sole images are not very adequate up to the critical Dean number to deduce whether the asymmetrical or symmetrical is superior in mixing.

Figure 10 presents a distinguishable comparison based on the visual data for Dean numbers above the critical Dean number so that the mixing results are clearer.

(a)

(i)

(ii)

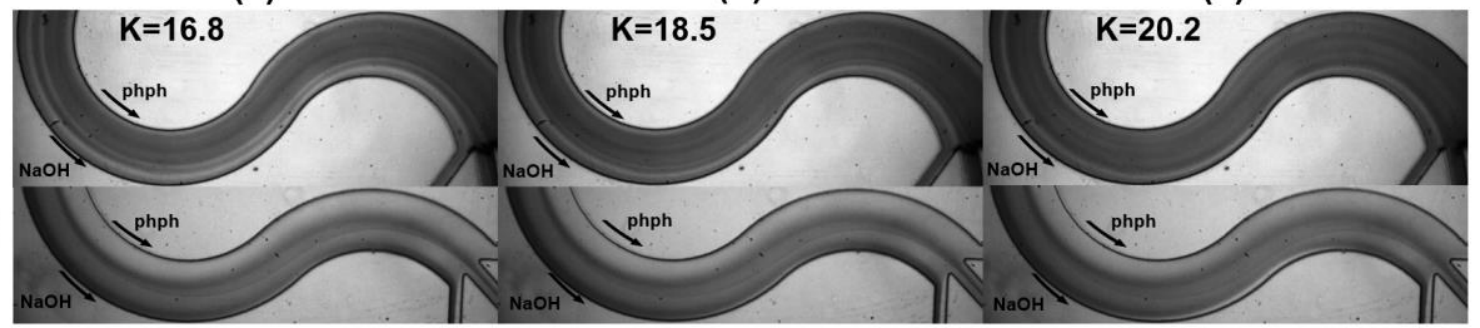

(d) (b)

(e) (c)

(f)

(i)

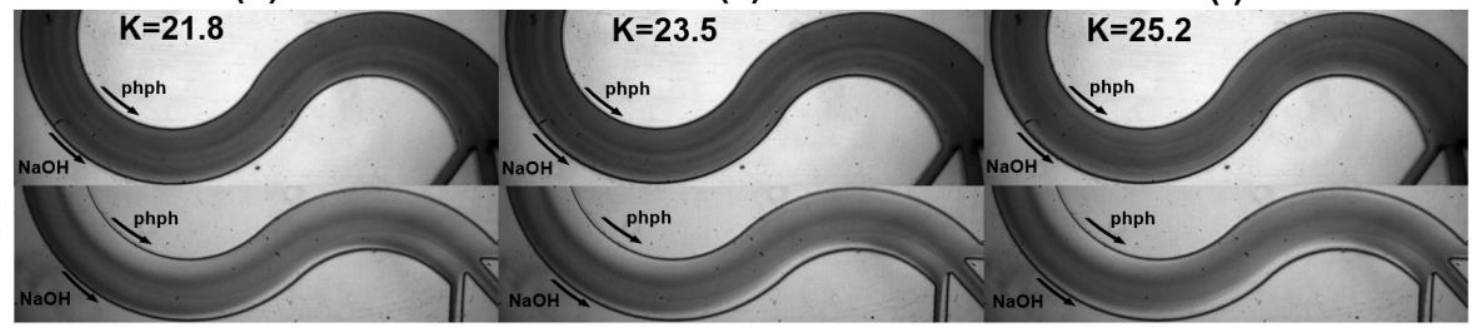

Figure 10: Mixing images of Phenolphthalein and $\mathrm{NaOH}$ aqueous solutions in: i) asymmetrical and ii) symmetrical micromixers for Dean numbers above the specified critical Dean number $(\mathrm{K}>16.8)$. The visualization was performed at the outlet region and the corresponding flow rates are a) $2000 \mu \mathrm{l} / \mathrm{min}$, b) $2200 \mu \mathrm{l} / \mathrm{min}$, c) $2400 \mu \mathrm{l} / \mathrm{min}$, d) 2600 $\mu \mathrm{l} / \mathrm{min}$, e) $2800 \mu \mathrm{l} / \mathrm{min}$ and f) $3000 \mu \mathrm{l} / \mathrm{min}$

Figure 10a demonstrates the visual data of both micromixers at the specified critical Dean number of $K=16.8$ determined according to mixing index data, where the mixing capability of the asymmetrical micromixer surpasses that of the symmetrical one. This is due to the only difference between these micromixers, which is the effect of the stretching and 
contracting motion ongoing throughout the asymmetrical micromixer. Both micromixers share the effect of two counter rotating Dean vortices, which emerge from the velocity gradient of the Poiseuille velocity profile combined with the velocity maxima shift due to curvilinearity and the redirection of these vortices at every turn. Since the only differing parameter between the asymmetrical and symmetrical micromixers is the recurrent widening and tightening of the microchannel walls between $450 \mu \mathrm{m}$ and $600 \mu \mathrm{m}$ inside the asymmetrical micromixer, it can be deduced that above the critical Dean number the superiority of the asymmetrical microchannel in mixing shown in Figures 10b-f results from this stretching and contracting motion of the Dean vortices.

Although both micromixers display a widespread mixing area throughout the horizontal axis at Dean numbers ranging from 18.5 to 25.2, a better mixing capability of the asymmetrical micromixer is evident due to its explicitly darker mixing region compared to the mixing region of the symmetrical one (Figures 10b-f). It should be noted that mixing at these flow rates is highly dominated by the chaotic advection since the corresponding Peclet number ranges from 162257 to 221260 . A combined analysis of visual results with the mixing index analysis, which is provided in Figure 11, is essential for a thorough evaluation of the results. The mixing index values were acquired from the line $(\mathrm{C}-\mathrm{C})$ near the outlet region for both micromixers (Figure 1) in order to be able to provide an analysis over a stabilized flow regime. 


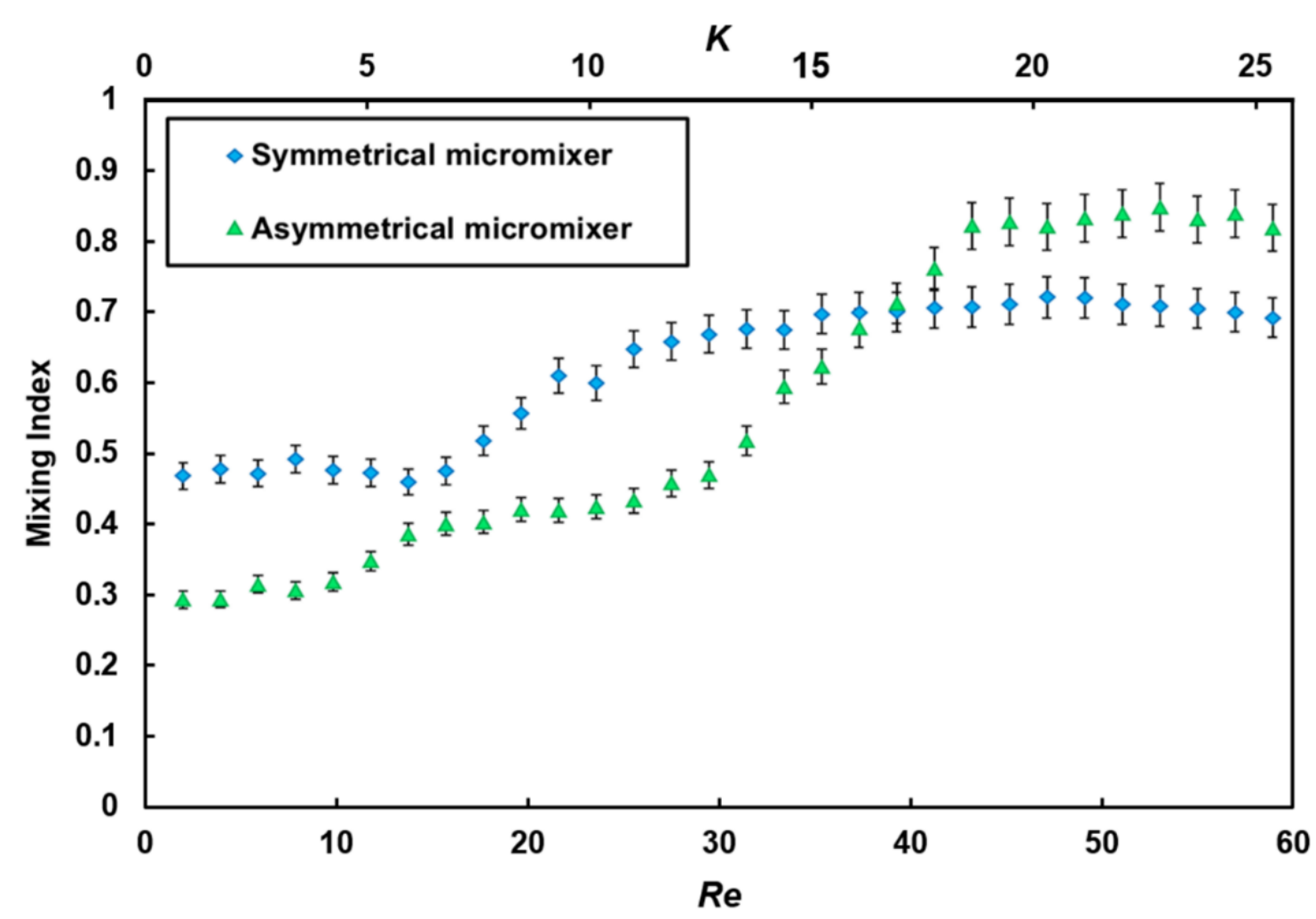

Figure 11: Mixing index as a function of Reynolds number (bottom x-axis) and Dean number (top x-axis) for symmetrical and asymmetrical micromixers

The given profiles propound the mixing index of both micromixers with respect to increasing $K$ and $R e$ for the ranges of 0.84 to 25.2 and 2 to 59, respectively. Considering Dean numbers, for $K<13.5$ the symmetrical microchannel proves itself to be superior to its asymmetrical counterpart with a maximum mixing index ratio of 1.5 at $K=11$. This result emerges from the inefficiency of a stretching and contracting motion at low flow rates since stretching vortices lose their strength while entering a turn with a widening wall. Even though the better mixing performance of the symmetrical micromixer is evident up to the critical Dean number, it can be seen that for $13.5<K<16.8$ there is a sharp increase in the mixing index of the asymmetrical microchannel indicating the strength of secondary flows even in relatively wider regions. Thus, the disturbance of the flow regime by this stretching and contracting motion starts to become beneficial. Above the designated threshold, the mixing performance of the asymmetrical micromixer surpasses its symmetrical counterpart. The maximum mixing index of 0.85 is obtained from the asymmetric micromixer at $K=22.7$ while for the same Dean number the mixing index of the symmetric micromixer is 0.71 . 
In addition, Figure 11 reveals that beyond a specific Dean number, both symmetrical and asymmetrical micromixers show a trend of stabilization in mixing efficiency. For the asymmetrical micromixer, this trend of stabilization starts around $K=18$, while the beginning of this stabilization trend manifests itself earlier $(K \cong 15)$ for the symmetrical micromixer. The mixing performance of the asymmetrical microchannel is capable of competing with curved planar micromixers having additional obstructions (Alam, Afzal, and Kim 2014).

\subsection{Numerical Results}

This work is mainly an experimental study rather than a numerical study. The simulations were performed in order to emphasise on how the velocity profile shift occurs beyond each inflection point due the curvilinearity and how the change in the velocity profile from A-A line to B-B line, which marks the relatively weakened velocity maxima, results in weakened Dean vortices.

The velocity profiles, which were obtained from flow simulations, in Figure 12 provides insight on how the velocity profile shift occurs after each inflection point due the curvilinearity.

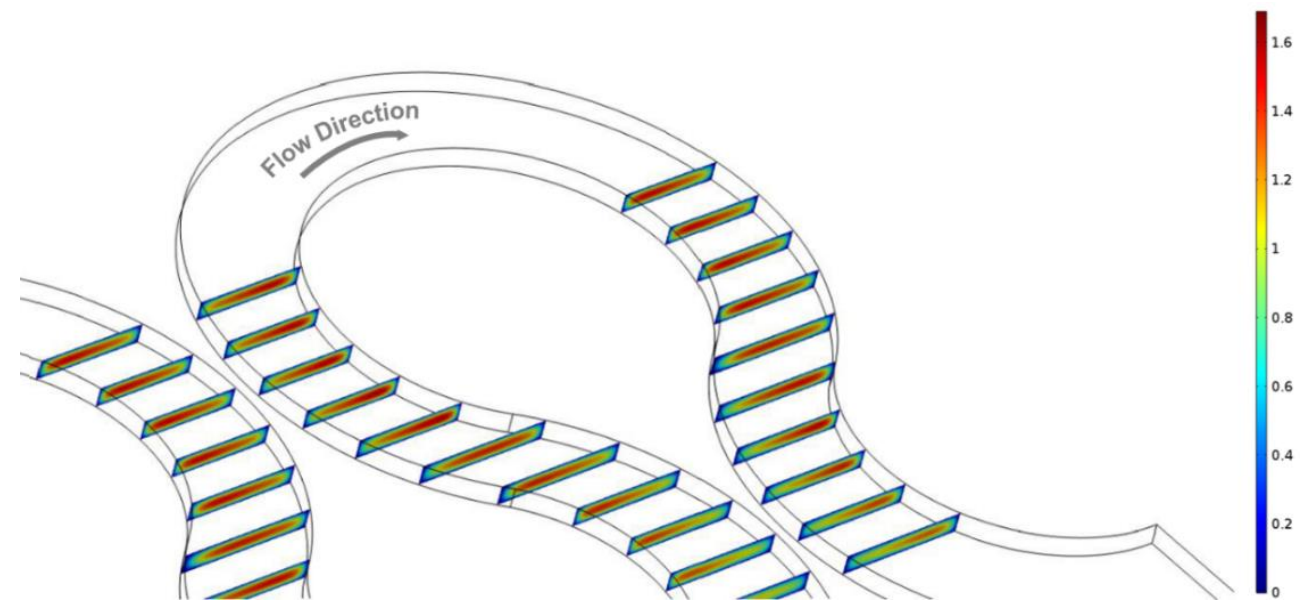

Figure 12: Velocity profiles of the asymmetrical microchannel near the outlet region showing the velocity profile shift after each inflection point at the flow rate of $2000 \mu \mathrm{l} / \mathrm{min}$, where the asymmetrical micromixer begins to outperform the symmetrical counterpart 
Additionally, the change in the velocity profile from $S_{2}$ line to $S_{1}$ line depicts the relatively higher velocity maxima, which results in stronger Dean vortices. Figure 13 shows the numerical results for better proving a better understanding of Dean vortices and velocity maxima shift.

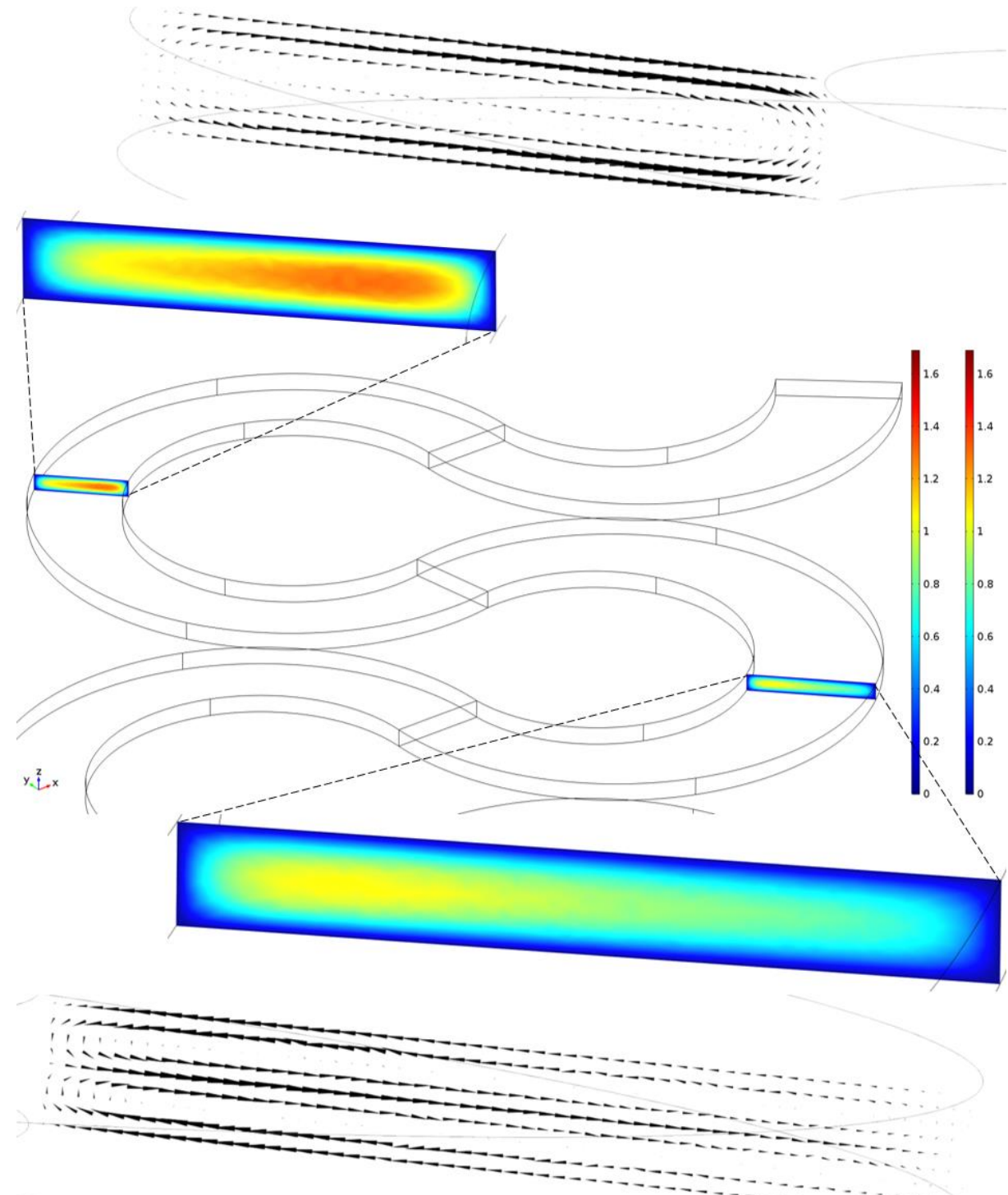

Figure 13: Cross-sectional velocity profile of the stretching region with a width of $450 \mu \mathrm{m}$ (upper) and the contraction region with a width of $600 \mu \mathrm{m}$ (lower) and their corresponding Dean vortices at the flow rate of $2000 \mu \mathrm{l} / \mathrm{min}$ 
These results are in agreement with the trends in the expected shift in the velocity profile of Poiseuille flows, which causes the formation of Dean vortices at the critical Dean number. From Figure 13, a detailed cross-sectional numerical velocity profile of the asymmetrical micromixer can be seen at herein before the specified $S_{1}$ line, which corresponds to a narrower width of $450 \mu \mathrm{m}$ and specified $S_{2}$ line, represents a wider width of $600 \mu \mathrm{m}$. Both velocity profiles at $K=16.8$ include the secondary flows, which results in Dean vortices combined with a more precise view of the velocity profile shift. It should be noted that the velocity profile taken at $S_{2}$ line, where the microchannel is wider, illustrates the weaker Dean vortices formation compared to the narrower $S_{1}$ region, which can be seen from the intensity of the vectors depicting the strength of the Dean vortices. This difference in strength is in compliance with the expressions of Reynolds and Dean number, where the velocity maxima hence the magnitudes of the velocity profile decrease due to the widening of the microchannel, and result in a smaller velocity gradient, which then generates less inertia on the fluid elements leading to weaker Dean vortices. The counter rotational motion of Dean vortices at C-C line (width of $450 \mu \mathrm{m}$ ) and D-D line (width of $600 \mu \mathrm{m}$ ) near the outlet is also provided with the velocity profiles in Figure 14. 


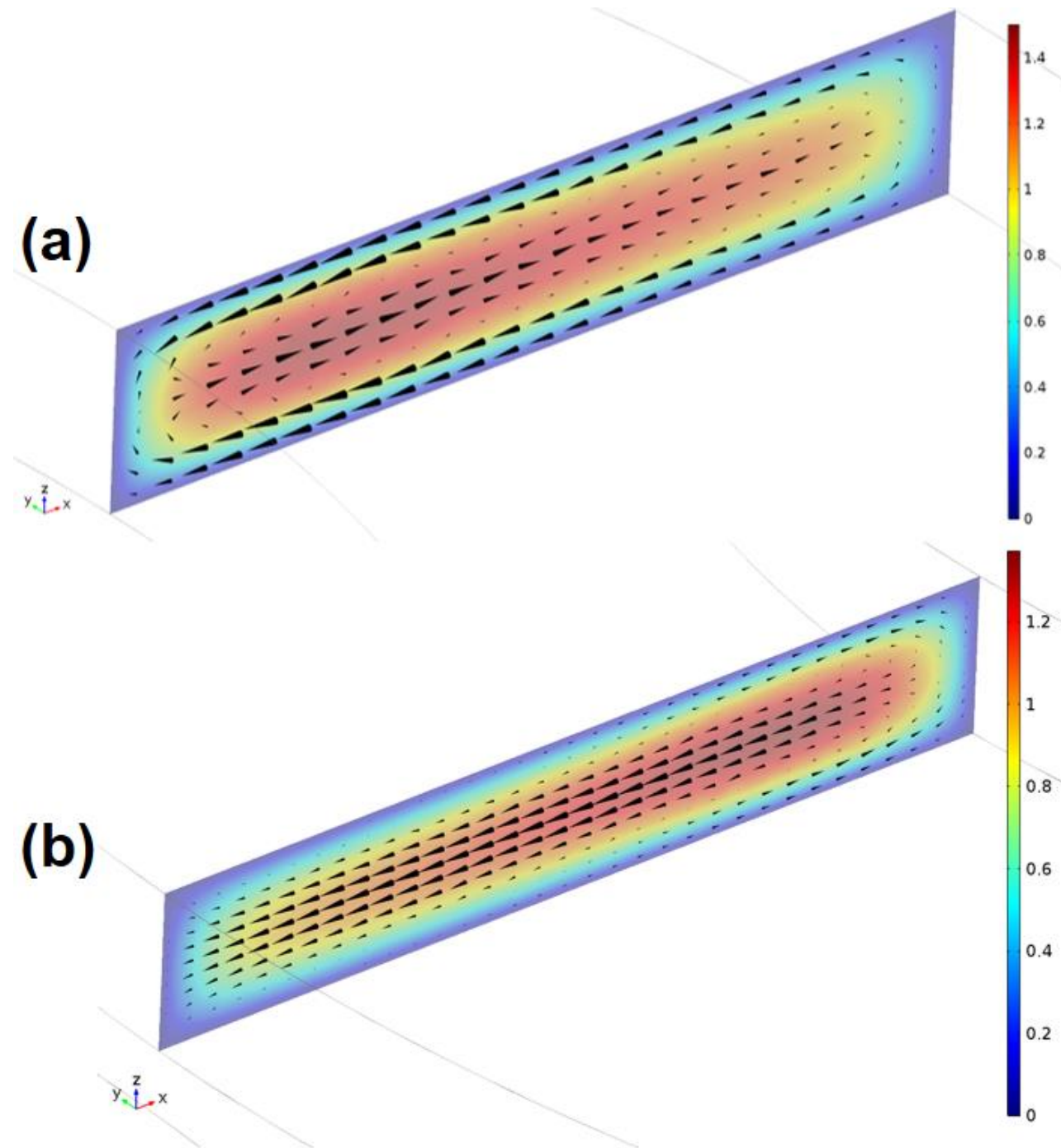

Figure 14: Cross-sectional velocity profile at regions: a) C-C line (width of $450 \mu \mathrm{m}$ ) and b) D-D line (width of $600 \mu \mathrm{m}$ ), showing Dean vortices at the flow rate of $2000 \mu \mathrm{l} / \mathrm{min}$, which is the critical pointi beyond which the asymmetrical micromixer outperforms the symmetrical one

Figures 13 and 14 also reveal the mixing enhancing effect of stretching and contracting motion in this study at high Dean numbers $(K \geq 16.8)$ by depicting the weaker Dean vortices formation of wider microchannels but also by pointing out that the superior mixing performance of the asymmetrical microchannel, which arises from the stretching and contracting of these vortices. From Figure 13, it can be deduced that the weakening of Dean 
vortices at the end of the wider turns has a negative effect on mixing. However, Figure 14 reveals that the vortices regain their power at narrower turns before entering to each wider turn. The resulting stretching and contracting motion enhances the mixing capability. These figures combined with the mixing index analysis prove that the effect of the stretching and contracting motion surpasses the negative effect of weaker and wider vortices at high Dean numbers $(\mathrm{K} \geq 16.8)$. 


\section{CHAPTER 4}

\section{CONCLUSION AND FUTURE WORK}




\subsection{Concluding Remarks}

In this dissertation, mixing performances of two curvilinear micromixers were investigated. Mixing index data combined with the visual data reveal the superiority of the symmetrical micromixer over the asymmetrical counterpart for the Dean number $K<16.8$. Beyond this threshold, the effect of stretching and contracting motion of two counter-rotating Dean vortices in the asymmetrical micromixer results in a higher mixing performance compared to the symmetrical one. In addition to comparison, the mixing indices of both micromixers are provided for a broad range of Reynolds numbers and Dean numbers based on the entrance, which range from 1.96 to 59 and from 0.84 to 25.2 , respectively.

Furthermore, the shift of the Poiseuille velocity profile at every turn towards the relative outer wall and Dean vortices are also illustrated via the numerical simulations. The purpose of this dissertation is to provide an insight into the effect of asymmetry in the width on mixing ability in order to assist the microfluidics community. This parametric study proves that the superiority of mixing for the given dimensions is dependent on operating conditions. To achieve a better mixing performance compared to the top mixing efficiency of 0.85 obtained from the asymmetrical micromixer in this parametric study, it can be suggested that the design can be further optimized through either obstacles, or the split and recombine method, which will be considered in future studies.

Micromixing is important for the preparation of pharmaceutical formulations, blending of different chemicals and drugs. Aggregations and segregations should be avoided during these procedures. In addition to chemical procedures such as chemical synthesis, polymerization, crystallization and extraction, micromixing procedures may be used in chemical content analyses using advanced detection systems (e.g. NMR, FTIR, or Raman spectroscopies). There are several applications in medicine as well. Many drugs come as crystals and require proper mixing and dissolution in physiological solutions (e.g. isotonic saline solutions). Micromixing systems can be used in DNA, RNA and protein analysis systems, enzymatic activity screens, single cell-based systems etc. Therefore, applications of

microfluidic systems include basic molecular biology and biomedicine research, drug discovery as well as clinical diagnosis and follow-up of various diseases. 


\subsection{Future Work}

In the near future, the aim will be to conduct experimental and numerical mixing analyses for various width asymmetry ratios. While this study provides the proof-of-concept

for enhanced mixing with asymmetry, an extensive numerical/experimental study will be conducted for the optimization of the asymmetry level. The data collected from the optimization of the asymmetry level will be used in new micromixer designs for the purpose of its integration into micro-total analysis systems (micro-TAS). 


\section{SELECTIVE BIBLIOGRAPHY}

1. Afzal, Arshad, and Kwang Yong Kim. 2012. "Passive Split and Recombination Micromixer with Convergent-Divergent Walls." Chemical Engineering Journal 203 (September 2012). Elsevier B.V.: 182-92. doi:10.1016/j.cej.2012.06.111.

2. Alam, Afroz, Arshad Afzal, and Kwang Yong Kim. 2014. "Mixing Performance of a Planar Micromixer with Circular Obstructions in a Curved Microchannel." Chemical Engineering Research and Design 92 (3). Institution of Chemical Engineers: 423-34. doi:10.1016/j.cherd.2013.09.008.

3. Aubin, J., D. F. Fletcher, and C. Xuereb. 2005. "Design of Micromixers Using CFD Modelling." Chemical Engineering Science 60 (8-9 SPEC. ISS.): 2503-16. doi:10.1016/j.ces.2004.11.043.

4. Beebe, David J, Glennys a Mensing, and Glenn M Walker. 2002. "Physics and Applications of Microfluidics in Biology." Annual Review of Biomedical Engineering 4: 261-86. doi:10.1146/annurev.bioeng.4.112601.125916.

5. Cha, Junghun, Jinseok Kim, Suk-Kyu Ryu, Jungyul Park, Yongwon Jeong, Sewan Park, Sukho Park, Hyeon Cheol Kim, and Kukjin Chun. 2006. "A Highly Efficient 3D Micromixer Using Soft PDMS Bonding." Journal of Micromechanics and Microengineering 16 (9): 1778-82. doi:10.1088/0960-1317/16/9/004.

6. Chen, Jyh Jian, Chun Huei Chen, and Shian Ruei Shie. 2011. "Optimal Designs of Staggered Dean Vortex Micromixers." International Journal of Molecular Sciences 12 (6): 3500-3524. doi:10.3390/ijms12063500.

7. Chen, Yu Tzu, Wei Feng Fang, Yen Cheng Liu, and Jing Tang Yang. 2011. "Analysis of Chaos and FRET Reaction in Split-and-Recombine Microreactors." Microfluidics and Nanofluidics 11 (3): 339-52. doi:10.1007/s10404-011-0803-8.

8. Cook, Kristina J. 2013. "Mixing Evaluation of a Passive Scaled-Up Serpentine Micromixer With Slanted Grooves." Journal of Fluids Engineering 135 (8): 081102. doi:10.1115/1.4024146.

9. DeMello, A J. 2006. "Control and Detection of Chemical Reactions in Microfluidic Systems.” Nature 442 (7101): 394-402. doi:10.1038/nature05062.

10. Fan, Liang-Liang, Xiao-Liang Zhu, Hong Zhao, Jiang Zhe, and Liang Zhao. 2017. "Rapid Microfluidic Mixer Utilizing Sharp Corner Structures." Microfluidics and Nanofluidics 21 (3). Springer Berlin Heidelberg: 36. doi:10.1007/s 10404-017-1874$\mathrm{y}$.

11. Gallé, Nicolas, and Victor Steinberg. 2016. "On-Chip Encapsulation via Chaotic Mixing." Microfluidics and Nanofluidics 20 (11). Springer Berlin Heidelberg: 1-10. doi:10.1007/s10404-016-1820-4.

12. Gobby, D, P Angeli, and A Gavriilidis. 2001. "Mixing Characteristics of T-Type Microfluidic Mixers." Journal of Micromechanics and Microengineering 11: 12632. doi:10.1088/0960-1317/11/2/307.

13. Hardt, S., K. S. Drese, V. Hessel, and F. Schönfeld. 2005. "Passive Micromixers for Applications in the Microreactor and MTAS Fields." Microfluidics and Nanofluidics 1 (2): 108-18. doi:10.1007/s10404-004-0029-0.

14. Hong, Chien-Chong, Jin-Woo Choi, and Chong H. Ahn. 2004. "A Novel In-Plane Passive Microfluidic Mixer with Modified Tesla Structures." Lab on a Chip 4 (2): 
109-13. doi:10.1039/B305892A.

15. Hosokawa, Kazuo, Teruo Fujii, and Isao Endo. 1999. "Droplet-Based Nano/Picoliter Mixer Using Hydrophobic Microcapillary Vent.” In IEEE Micro Electro Mechanical Systems, 388-93. doi:10.1109/MEMSYS.1999.746860.

16. Jeong, Gi Seok, Seok Chung, Chang-Beom Kim, and Sang-Hoon Lee. 2010. "Applications of Micromixing Technology." The Analyst 135 (3): 460-73. doi:10.1039/b921430e.

17. Jiang, F., K. S. Drese, S. Hardt, M. Küpper, and F. Schönfeld. 2004. "Helical Flows and Chaotic Mixing in Curved Micro Channels." AIChE Journal 50 (9): 22972305. doi:10.1002/aic.10188.

18. Kang, Tae Gon, Mrityunjay K. Singh, Patrick D. Anderson, and Han E H Meijer. 2009. "A Chaotic Serpentine Mixer Efficient in the Creeping Flow Regime: From Design Concept to Optimization." Microfluidics and Nanofluidics 7 (6): 783-94. doi:10.1007/s10404-009-0437-2.

19. Kang, Tae Gon, Mrityunjay K. Singh, Tai Hun Kwon, and Patrick D. Anderson. 2008. "Chaotic Mixing Using Periodic and Aperiodic Sequences of Mixing Protocols in a Micromixer." Microfluidics and Nanofluidics 4 (6): 589-99. doi:10.1007/s10404-007-0206-z.

20. Kim, Dong Sung, In Hwan Lee, Tai Hun Kwon, and Dong-Woo Cho. 2004. "A Barrier Embedded Kenics Micromixer." Journal of Micromechanics and Microengineering 14 (10): 1294-1301. doi:10.1088/0960-1317/14/10/002.

21. Koch, M, H Witt, a G R Evans, and A Brunnschweiler. 1999. "Improved Characterisation Technique for Micromixers" 156. doi:10.1088/0960-1317/9/2/312.

22. Kockmann, Norbert, Thomas Kiefer, Michael Engler, and Peter Woias. 2006. "Convective Mixing and Chemical Reactions in Microchannels with High Flow Rates." Sensors and Actuators, B: Chemical 117 (2): 495-508. doi:10.1016/j.snb.2006.01.004.

23. Lee, Chia Yen, Wen Teng Wang, Chan Chiung Liu, and Lung Ming Fu. 2016. "Passive Mixers in Microfluidic Systems: A Review." Chemical Engineering Journal 288. Elsevier B.V.: 146-60. doi:10.1016/j.cej.2015.10.122.

24. Liu, Changgeng, Zhong-geng Ling, Kun Lian, Jost Goettert, Josef Hormes, and Baton Rouge. 2006. "An Injection Micromixer Fabricated by Improved SU-8 Processing for Biochemical Microfluidic Systems.” In Spie, edited by Ian Papautsky and Wanjun Wang, 6112:1-7. San Jose, CA. doi:10.1117/12.646049.

25. Livak-Dahl, Eric, Irene Sinn, and Mark Burns. 2011. "Microfluidic Chemical Analysis Systems." Annu. Rev. Chem. Biomol. Eng. 2: 325-53. doi:10.1146/annurev-chembioeng-061010-114215.

26. Marques, Marco P C, and Pedro Fernandes. 2011. "Microfluidic Devices: Useful Tools for Bioprocess Intensification.” Molecules 16 (10): 8368-8401. doi:10.3390/molecules16108368.

27. Mengeaud, Virginie, Jacques Josserand, and Hubert H. Girault. 2002. "Mixing Processes in a Zigzag Microchannel: Finite Element Simulations and Optical Study." Analytical Chemistry 74 (16): 4279-86. doi:10.1021/ac025642e.

28. Nguyen, Nam-Trung, and Zhigang Wu. 2005. "Micromixers - a Review." Journal of Micromechanics and Microengineering 15 (2): R1-16. doi:10.1088/09601317/15/2/R01.

29. Park, Jang Min, Dong Sung Kim, Tae Gon Kang, and Tai Hun Kwon. 2008. 
"Improved Serpentine Laminating Micromixer with Enhanced Local Advection." Microfluidics and Nanofluidics 4 (6): 513-23. doi:10.1007/s10404-007-0208-x.

30. Schwesinger, Norbert, Thomas Frank, and Helmut Wurmus. 1999. "A Modular Microfluid System with an Integrated Micromixer." Journal of Micromechanics and Microengineering 6 (1): 99-102. doi:10.1088/0960-1317/6/1/023.

31. Singh, Mrityunjay K., Tae Gon Kang, Han E H Meijer, and Patrick D. Anderson. 2008. "The Mapping Method as a Toolbox to Analyze, Design, and Optimize Micromixers." Microfluidics and Nanofluidics 5 (3): 313-25. doi:10.1007/s10404007-0251-7.

32. Stroock, Abraham D., and Gregory J. McGraw. 2004. "Investigation of the Staggered Herringbone Mixer with a Simple Analytical Model." Philosophical Transactions of the Royal Society of London A: Mathematical, Physical and Engineering Sciences 362 (1818): 971-86. doi:10.1098/rsta.2003.1357.

33. Sudarsan, Arjun P, and Victor M Ugaz. 2006. "Multivortex Micromixing." In Proceedings of the National Academy of Sciences of the United States of America, 103:7228-33. doi:10.1073/pnas.0507976103.

34. Tofteberg, Terje, MacIej Skolimowski, Erik Andreassen, and Oliver Geschke. 2010. "A Novel Passive Micromixer: Lamination in a Planar Channel System." Microfluidics and Nanofluidics 8 (2): 209-15. doi:10.1007/s10404-009-0456-z.

35. Tung, Kai Yang, and Jing Tang Yang. 2008. "Analysis of a Chaotic Micromixer by Novel Methods of Particle Tracking and FRET." Microfluidics and Nanofluidics 5 (6): 749-59. doi:10.1007/s10404-008-0287-3.

36. Voldman, Joel, Martha L. Gray, and Martin A. Schmidt. 2000. "Integrated Liquid Mixer/Valve." Journal of Microelectromechanical Systems 9 (3): 295-302. doi:10.1109/84.870054.

37. Ward, Kevin, and Z. Hugh Fan. 2015. "Mixing in Microfluidic Devices and Enhancement Methods." Journal of Micromechanics and Microengineering 25 (9). IOP Publishing: 094001. doi:10.1088/0960-1317/25/9/094001.

38. Xia, H M, C Shu, S Y M Wan, and Y T Chew. 2006. "Influence of the Reynolds Number on Chaotic Mixing in a Spatially Periodic Micromixer and Its Characterization Using Dynamical System Techniques.” Journal of Micromechanics and Microengineering 16 (1): 53-61. doi:10.1088/09601317/16/1/008.

39. Xia, H M, S Y M Wan, C Shu, and Y T Chew. 2005. "Chaotic Micromixers Using Two-Layer Crossing Channels to Exhibit Fast Mixing at Low Reynolds Numbers." Lab on a Chip 5 (7): 748-55. doi:10.1039/b502031j.

40. Yi, Mingqiang, and Haim H. Bau. 2003. "The Kinematics of Bend-Induced Mixing in Micro-Conduits." International Journal of Heat and Fluid Flow 24 (5): 645-56. doi:10.1016/S0142-727X(03)00026-2.

41. Zhang, Yanrong, Yandong Hu, and Huiying Wu. 2012. "Design and Simulation of Passive Micromixers Based on Capillary." Microfluidics and Nanofluidics 13 (5): 809-18. doi:10.1007/s10404-012-1002-y. 